\title{
Benoît RossignOL
}

Université Paris 1 Panthéon-Sorbonne / centre Gustave Glotz

\author{
" Les préfets du prétoire de Marc Aurèle » \\ Cahiers Glotz, XVIII, 2007, p. 141-177
}

\section{Summary / Résumé}

From AD 161 to 180 we know 7 Praetorian prefects, we survey their careers and their functions at the emperor's side. Under Marcus Aurelius the Praetorian prefects had an important role on campaign. If the emperor sought to appoint prefects who were skilled in military affairs, he sought also other competences : prefects were carefully chosen in the top of the equites. Siting in the consilium principis, the prefects had also activities which were not necessarily connected with their duties as the Guard commander. The position of these prefects was influential, but it must not be overstated, to understand their office we must replace it in its context, the emperor's entourage.

De 161 à 180 nous connaissons sept préfets du prétoire. Nous analysons leurs carrières et leurs fonctions aux côtés de l'empereur. Durant le règne de Marc Aurèle les préfets du prétoire jouèrent un rôle important dans les campagnes militaires. Si l'empereur rechercha des hommes expérimentés dans le domaine de la guerre, il cherchait aussi d'autres compétences : les préfets étaient soigneusement choisis au sommet de l'ordre équestre. Siégeant au consilium principis, les préfets avaient d'autres activités qui n'étaient pas nécessairement liées à leur fonction de chef de la garde. Ils étaient des hommes d'influence, mais ce trait ne doit pas être exagéré, on ne peut comprendre leur charge qu'en la replaçant dans son contexte, l'entourage de l'empereur. 
BENOIT ROSSIGNOL

LES PRÉFETS DU PRÉTOIRE DE MARC AURÈLE *

Le règne de Marc Aurèle se caractérise par de graves difficultés pour l'Empire, dont les prodromes apparaissent dès les dernières années du règne d'Antonin le Pieux. Des guerres longues et difficiles se succèdent et mettent durement à l'épreuve l'administration impériale. Cette situation n'a pas manqué d'avoir des effets sur le personnel dirigeant et logiquement sur les personnages clés que sont les préfets du prétoire ainsi que sur leurs domaines d'intervention. Nous nous proposons ici d'analyser leur place aux côtés de l'empereur et au sein du personnel dirigeant dans une période militairement difficile où la légitimité impériale est cependant forte. Pour cela nous établirons d'abord la liste et la chronologie des titulaires du poste de 161 à 180, puis examinerons successivement chacune de leur carrière. Nous considérerons ensuite quelle a pu être leur place aux côtés du prince à partir des représentations offertes par nos sources, littéraires, épigraphiques mais aussi figuratives. Le contexte de la période exige de considérer de près leur rôle et leur place dans les armées en campagne, les commandements qui leur ont été confiés, leur rôle de conseiller militaire du prince. Leur fonction ne s'arrêtait toutefois pas là et il faudra examiner ce que nous pouvons savoir, pour la période considérée, de leur rôle administratif et judiciaire avant d'essayer d'éclairer, en conclusion, les choix du prince et leurs conséquences au sein des sommets du pouvoir.

\section{TITULAIRES DU POSTE}

\section{Chronologie}

Il n’y eut pas, du point de vue de la préfecture du prétoire, solution de continuité entre le règne d'Antonin le Pieux et celui, conjoint, de Marc Aurèle et de Lucius Vérus. Après la charge exceptionnellement longue de Gavius Maximus, et celle plus courte de Tattius Maximus, Antonin, dans les derniers mois de son règne, avait réinstauré un exercice collégial de la fonction. Le texte de l’Histoire Auguste, qui témoigne de cette succession,

\footnotetext{
* Une grande partie de l'étude qui suit est issue d'un chapitre de notre thèse de doctorat, Études sur l'empire romain en guerre durant le règne de Marc Aurèle (161-180), thèse de doctorat soutenue à l'université Paris 1 Panthéon-Sorbonne sous la direction de M. le professeur Michel Christol, novembre 2004, 1393 p. dactylographiée et d'une communication présentée le 12 juin 2004 à l'occasion de la table ronde du programme Empire en mutation de l'UMR 8585. Nous voulons remercier ici $\mathrm{M}$. Michel Christol pour sa relecture attentive et ses suggestions précieuses, nous gardons cependant la responsabilité d’éventuelles erreurs.
} 
n'a pas conservé de manière rigoureuse les noms des deux préfets $^{1}$ : Furius Victorinus et Cornelius Repentinus. Cependant ils se restituent sans difficulté, et sont associés sur une inscription romaine: il s'agit de l'épitaphe d'un prétorien qui mourut en 167, et était entré dans les troupes du prétoire, durant les fonctions de ces deux préfets, en $163^{2}$.

Ces deux personnages furent les garants, au printemps 161, de la passation de pouvoir. C'est en leur présence qu'Antonin, sur son lit de mort, réaffirma qu'il confiait l'empire à son gendre, Marc Aurèle ${ }^{3}$. Marc s’adjoignit Lucius Vérus comme collègue. Il faut sans doute imaginer que les deux préfets furent présents aux côtés des deux empereurs quand ces derniers se présentèrent au camp des prétorien et $\mathrm{y}$ annoncèrent un donativum de 20000 sesterces par soldat ${ }^{4}$. Par la suite Furius Victorinus participa à la guerre parthique en Orient, aux côtés de Lucius Vérus ${ }^{5}$. Les deux préfets restèrent en fonction plusieurs années. Cornelius Repentinus au moins jusqu'en 165, comme en témoigne son cursus retrouvé à Pouzzoles ${ }^{6}$. On considère en général que sa charge se termina vers 167 . Son collègue Furius Victorinus mourut en fonction vers la fin du printemps 168, alors qu'il accompagnait les deux empereurs vers les provinces frontières menacées par les barbares ${ }^{7}$ et alors que la peste touchait durement l'Italie et l'armée romaine.

Victorinus fut remplacé par Bassaeus Rufus, qui était parti, très peu de temps auparavant, diriger l'Égypte, et la quitta au plus tard au printemps $169^{8}$. La garde de l'empereur était donc dirigée par un nouveau couple de préfets dont les noms se retrouvent sur la célèbre inscription de Saepinum ${ }^{9}$ : Bassaeus Rufus et Macrinius Vindex. Ce dernier étant lui aussi préfet depuis peu, au plus tôt depuis le départ de Repentinus. Cependant, il faut observer que sur l'inscription de Saepinum son nom vient toujours après celui de Bassaeus Rufus, il faudrait donc penser qu'il avait moins d’ancienneté dans le poste que ce dernier. Quoi qu'il en soit, les deux préfets accompagnèrent à nouveau l'empereur vers les régions danubiennes où les menaces étaient

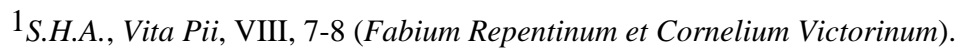

${ }^{2}$ Not. Scavi 1915, 39 n 3 (AE 1916, n47) (Rome).

3 S.H.A., Vita Pii, XII, 5 et Vita Marci, VII, 3, qui précise que les Amis d’Antonin étaient aussi présents.

${ }^{4}$ S.H.A., Vita Marci, VII, 9. L’association de Vérus au pouvoir impérial devait nécessairement concerner les préfets. Selon l'Histoire Auguste, Vita Veri, III, 5, avant la mort d'Antonin, Vérus voyageait dans la voiture d'un des préfets du prétoire, mais nous ne savons s'il faut vraiment accorder du crédit à ce détail qui peut être un anachronisme. Cf. J.-P. Callu, "Verus avant Verus", Historiae Augustae Colloquia, Nova Series I, Colloquium Parisinum 1990 (désormais H.A.C. 1990), Macerata, 1991, p. 119-120.

${ }^{5}$ CIL, VI, 41143, dernière édition de CIL XIV, *440 ; CIL V, *648 ; CIL VI, *1937 ; Huelsen, Ausonia, II, 1907, pp. 67-76 (AE 1907, n¹52) (ILS 9002) ; CIL VI, 39440a.

6 CIL X, 1856 et G. Camodeca, « La carriera del prefetto del pretorio Sex. Cornelius Repentinus in una nuova iscrizione puteolana », Puteoli, III, 1979, p. 41-76 (AE 1980, n 235) ; Id., « La carriera del prefetto del pretorio Sex. Cornelius Repentinus in una iscrizione puteolana », ZPE, 43, 1981, p. 43-56.

7 S.H.A., Vita Marci, XIV, 5.

8 Cf. G. Bastianini, « Lista dei prefetti d’Egitto dal 30a al 299p. », ZPE, 17, 1975, p. 297.

${ }^{9}$ CIL IX, 2438 = 4916 (Italie, région IV, Saepinum) avec le gentilice de Vindex orthographié Macrinus.
} 
peut-être plus fortes que jamais. Macrinius Vindex mourut au combat en 172 au plus tard ${ }^{10}$.

Nous ne savons pas si Macrinius trouva immédiatement un successeur. Si l'on en croit l'Histoire Auguste, Marc Aurèle, à un certain moment de son règne, semble avoir eu du mal à désigner un préfet du prétoire, regrettant de ne pas pouvoir nommer Pertinax, entré au sénat ${ }^{11}$. En 173, alors que Marc Aurèle se trouve à Sirmium, lors du procès d'Hérode Atticus, Bassaeus Rufus est à ses côtés et intervient dans le procès ${ }^{12}$. Nous ne pouvons dire s'il était alors seul à exercer sa charge. Il l'exerçait probablement encore en 177, puisqu'à cette date, et plus précisément le 6 juillet de cette année, il faisait partie du conseil de l'empereur, comme nous l'a appris la tabula Banasitana $^{13}$. Il a aussi été avancé qu'à cette date, Bassaeus Rufus avait reçu les ornamenta consularia et pris sa retraite ${ }^{14}$. Quoi qu'il en soit, son nom sur ce document est suivi de celui de P. Taruttienus Paternus. La logique hiérarchique de cette liste de nom implique que Paternus ait été lui aussi préfet du prétoire ${ }^{15}$. Le nom de ce dernier est en effet suivi du nom, effacé, de Tigidius Perennis vraisemblablement alors préfet de l'annone comme l'a suggéré H.-G. Pflaum ${ }^{16}$. Puis venait le nom de Cervidius Scaevola qui, deux ans auparavant, était préfet des vigiles ${ }^{17}$. W. Seston et M. Euzennat, estimant que Bassaeus Rufus venait de se retirer de sa charge, considérèrent que Paternus était alors préfet du prétoire avec Perennis ${ }^{18}$. Il nous semble que 177 est une date très haute pour placer les débuts de la préfecture du prétoire de Perennis, d'autant plus que selon Hérodien il n’obtint ce poste qu'après la mort de Marc Aurèle ${ }^{19}$. Le récit de Dion signale aussi qu'il dirigea les prétoriens après Paternus ce qui peut laisser

10 Dion Cassius, LXXI, 3, 5 (Xiphilin, 259, 26).

11 S.H.A., Vita Pertinacis, II, 9.

12 Philostrate, Vie des sophistes, II, 1, 11 (561) (éd. Wright, p. 171). Cf. F. Millar, The Emperor in the Roman World ${ }^{2}$, Londres, 1992, pp. 4-7. Nous ne comprenons pas pourquoi M. Absil, Les préfets du prétoire d'Auguste à Commode, Paris, 1997, p. 74 pense que Bassaeus prononça le verdict.

13 IAM, 94 ; Cf. H.-G. Pflaum, Les carrières procuratoriennes équestres sous le haut-empire romain, Supplément, (désormais Suppl.), 1982, p. 48.

14 CIL VI, 1599 + 31828 (ILS 1326) ; CIL VI, 41141. Cf. W. Seston et M. Euzennat, « Un dossier de la chancellerie romaine : la tabula Banasitana. Étude de diplomatique », CRAI, 1971 p. 486 désormais W. Seston, Scripta Varia, Rome, 1980, p. 103.

15 Très prudent F. Millar, The Emperor in the Roman World ${ }^{2}$, Londres, 1992, p. 130 ne s'avance pas sur la question. La préfecture de Paternus nous semble incontestable en 177. Sur l'interprétation de cette liste: M. Christol, «L'ascension de l'ordre équestre : un thème historiographique et sa réalité », in S. Demougin, H. Devijver, M.-Th. Raepsaet-Charlier dir., L'ordre équestre, Histoire d'une aristocratie (IIe siècle av. J.C. - IIIe siècle ap. J.C.), Rome, 1999, p. 622.

16 H.-G. Pflaum, «La valeur de la source inspiratrice de la vita Hadriani et de la vita Marci Antonini à la lumière des personnalités contemporaines nommément citées », B.H.A.C. 1968-1969, Bonn, 1970, p. 217-218 ; Id., « La valeur de l'information historique de la Vita Commodi à la lumière des personnages nommément cités par le biographe », B.H.A.C. 1970, Bonn, 1972, p. 205 ; Id., « La préfecture de l'annone (à propos d'un ouvrage récent) », RHD, 56, 1978, p. 68-69 et Suppl., 1982, p. 48 ; Perennis n'est pas retenu dans la liste dressée par H. Pavis d’Escurac, La préfecture de l'annone service administratif impérial d'Auguste à Constantin, Rome, 1976, p. 45-46.

17 CIL XIV, 4502 (ILS 2164) ; cf. M. Sablayrolles, Libertinus Miles, Rome, 1996, p. 489, nº 16.

18 W. Seston et M. Euzennat, op. cit.

19 Hérodien, I, 8, 1. 
penser que l'essentiel de sa fonction se déroula sous Commode ${ }^{20}$. Même si Hérodien n'est pas nécessairement fiable à ce propos, l'hypothèse de H.G. Pflaum nous semble correspondre à un rythme d'avancement plus vraisemblable.

Marc Aurèle, en 178, quitta à nouveau Rome pour les régions danubiennes. Paternus reçut le commandement de troupes nombreuses. Placé à leur tête il remporta en 179 une brillante et importante victoire qui valut à l'empereur sa dixième salutation ${ }^{21}$. Moins d'un an plus tard, il se retrouvait dans la position qui avait été celle de Furius Victorinus et Cornelius Repentinus en 161 : devoir assurer la transition entre deux règnes. Selon Hérodien, nous l'avons vu, Commode lui adjoignit Tigidius Perennis comme collègue, mais il n'est pas impossible que ce dernier ait été nommé par Marc Aurèle à l'extrême fin de son règne. Perennis et Paternus ne s'entendaient visiblement pas. Une profonde rivalité semble avoir existé entre eux. Rapidement, la conjuration de Lucilla fut l'occasion de la chute de Paternus, orchestrée par Perennis. Taruttienus fut admis parmi les consulaires et dut quitter sa charge. Puis il fut accusé de complot et éliminé22. Après trois ans de pouvoir apparemment sans partage, ce fut au tour de Perennis d'être victime des luttes politiques du règne ${ }^{23}$.

Il reste à évoquer le cas d'un personnage dont la fonction est très mal datée, mais que l'on place en général sous Marc Aurèle ${ }^{24}$ : il s'agit de T. Flavius Constans ${ }^{25}$. Il nous est connu par une inscription de Cologne, qui peut être datée de la période allant du règne d'Hadrien à celui de Marc Aurèle ${ }^{26}$. Il est identifié au procurateur homonyme de Dacie inférieure de 138, connu par deux inscriptions très bien datées ${ }^{27}$. À quelle date pouvonsnous placer un autre préfet dans notre chronologie ? Deux solutions s'offrent à nous. Tout d'abord de 165 à 168, entre Repentinus et Vindex, à supposer que Repentinus n'ait pas exercé sa fonction après 165, et ensuite de 172 à 177 au plus tard, comme collègue de Bassaeus Rufus après la mort de Vindex et avant l'entrée en charge de Paternus ${ }^{28}$. Avant la publication des derniers fragments de l'inscription de Pouzzoles dédiée à Repentinus,

20 Dion Cassius, LXXII, 9 et voir LXXII, 5.

21 Dion Cassius, LXXI, 33, 3-4 (Xiphilin, 267).

22 S.H.A., Vita Commodi, IV, 1-9 et Dion Cassius, LXXII, 5.

23 Dion Cassius, LXXII, 9-10 (voir P.A. Brunt, « The Fall of Perennis : Dio-Xiphilinus 72.9.2 », The Classical Quarterly, N.S., 23, 1, 1973, p. 172-177) ; Hérodien, I, 9 ; S.H.A., Vita Commodi, VI, $1-3$ et VIII, 1.

24 Ainsi W. Meyer, L'administration de la province romaine de Belgique, Bruges, 1964, pp. $77-$ 78 ; A.R. Birley, Marcus Aurelius, Londres, 1966, p. 178 ; M. Absil, op. cit., p. 181, n 42. L'hypothèse remonte à Poppelreuter, Römisch-Germanisches Korrespondenzbl., III, 1910, p. 2 sq. (n.v.) $\left(A E 1910, \mathrm{n}^{\circ} 67\right)$.

$25 P^{2}$ F 247 ; CPE n 149.

26 CIL XIII, 12057 ; B. et H. Galsterer, Die Römischen Steininschriften aus Köln, Cologne, 1975, n¹46. Cf. W. Eck, "Ein Kölner in Rom ? ", in V.A. Rieche, H.J. Schalles, M. Zelle, Grabung Forschung Präsentation, Festschrift Gundolf Precht, Mayence, 2002, p. 37-42.

27 CIL III, 12601=13793-13794 ; IDR II, 575 et CIL III, 13795 (ILS 8909) ; IDR II, 587.

28 M. Absil, op. cit., p. 24 ne tient visiblement pas compte des interprétations qui ont été proposées pour la Tabula Banasitana, il place Constans entre Bassaeus Rufus et Paternus soit en 177179, quarante ans après sa procuratèle centenaire : Constans aurait alors approché des soixante-dix ans ! Cela ne nous semble pas acceptable. 
Constans était en général placé à la tête du prétoire vers 163-165. C’est supposer près de trente ans de carrière entre sa procuratèle centenaire et sa préfecture, ce qui peut être normal ${ }^{29}$. Retenir la seconde hypothèse allonge considérablement le délai. D’autant plus que Constans aurait succédé à Furius Victorinus bien que plus ancien dans la carrière. Il faudrait donc placer Constans entre Cornelius Repentinus et Macrinius Vindex, pour un temps de fonction très bref vers 168. Mais il n'est peut-être pas non plus impossible de voir en lui un éphémère collègue de Gavius Maximus vers le milieu des années 150 : on ne peut donc pas lui assigner une chronologie assurée $^{30}$. Les carrières de ces différents préfets sont très inégalement connues $^{31}$ et ont été parfois très souvent commentées, en particulier pour celles qui sont les plus complètes. Nous rappellerons ici les traits essentiels de ces divers parcours afin de les caractériser et peut-être de comprendre la logique des choix du prince.

\section{Sex. Cornelius Quirina Repentinus signo Contuccius}

La carrière de ce personnage nous est mieux connue depuis la publication de nouveaux fragments de l'immense inscription qui lui était dédiée à Pouzzoles $^{32}$. Sans doute originaire de Simitthu en Afrique ${ }^{33}$, il débuta en tant qu'avocat du fisc, effectua une procuratèle centenaire, peut-être celle du vingtième des héritages selon Giuseppe Camodeca ${ }^{34}$, puis fut $a b$ epistulis d'Antonin, schéma de carrière que l'on retrouve pour son successeur à ce dernier poste, Caecilius Crescens Volusianus ${ }^{35}$. Si l'on en croit l'Histoire Auguste, une rumeur infamante prétendait que Repentinus avait obtenu sa préfecture grâce aux intrigues de la concubine de l'empereur ${ }^{36}$, que l'on peut identifier à Galeria Lysistrate ${ }^{37}$. Nous retrouvons cet administrateur influent parmi les correspondants de Fronton qui lui avait recommandé un Fabianus dont nous ignorons tout ${ }^{38}$. Il n'était sans doute pas mauvais de faire partie de l'entourage du préfet Cornelius Repentinus: son a commentariis fit une

29 H.-G. Pflaum, «Compte-rendu de W. Meyers, L'administration de la province romaine de Belgique », Gnomon, 37, 1965, p. 390 désormais Scripta Varia II, Paris, 1981, p. 78.

30 Voir G. Camodeca, « Carriera », 1981, cit. supra, p. 49.

31 La remarque vaut en général pour tous les préfets du prétoire, le règne de Marc Aurèle étant, finalement, de ce point de vue l'un des mieux documentés. Cf. M. Durry, Les cohortes prétoriennes, Paris, 1938, p. 159-163 ; M. Absil, op. cit., p. 39-43 reste peu précis et déçoit. Plus généralement sur les grandes préfectures voir R. Sablayrolles in S. Demougin, H. Devijver, M.-Th. Raepsaet-Charlier dir., L'ordre équestre, Histoire d'une aristocratie (IIe siècle av. J.C. - IIIe siècle ap. J.C.), Rome, 1999, p. 351-389.

32 Cf. PIR ${ }^{2}$ C 1428 ; G. Camodeca, « Carriera », 1979, cit. supra et « Carriera », 1981, cit. supra. Références et bibliographie dans : M. Absil, op. cit., p. 175-176, n³8.

33 G. Camodeca, «Carriera », 1981, cit. supra p. 44-45 ; S. Demougin, n. 355 dans Fronton, Correspondance, tr. fr. de P. Fleury, Paris, 2003, p. 308. M. Absil, op. cit., p. 28 situe ses origines à Pouzzoles ce qui est impossible puisqu'il est inscrit dans la tribu Quirina.

34 G. Camodeca, « Carriera », 1981, cit. supra, p. 53.

35 CIL VIII, 1174 (ILS 1451); cf. CPE, p. 337-339, nº 142.

36 S.H.A., Vita Pii, VIII, 9.

37 CIL VI, 8972 (ILS 1836).

38 Fronton, Ad Amicos, II, 4 (cf. P. Fleury, op. cit.). 
belle carrière équestre qui le mena au moins jusqu'au poste d'ab epistulis latinis avant de lui permettre d'entrer au sénat parmi les anciens préteurs ${ }^{39}$.

Si son collègue Victorinus partit pour l'Orient affronter les Parthes, Repentinus resta sans doute à Rome aux côtés de Marc Aurèle : il est vrai que son profil n'est pas militaire. Il obtint les ornements consulaires ${ }^{40}$. Nous ne savons pas exactement quand se termina sa charge. En tout cas, le sort de sa famille prospéra: un Cornelius Repentinus épousa la fille de Didius Iulianus et fut son éphémère préfet de la Ville en $193^{41}$.

\section{T. Furius L(ucii) filius Palatina Victorinus}

La carrière de Victorinus nous est connue dans son ensemble, mais plusieurs de ses détails posent problème et bien des incertitudes demeurent ${ }^{42}$. Son cursus figurait sur une inscription dont le texte passa longtemps pour faux avant d'être réhabilité au début du siècle dernier : il ne nous a pas été transmis cependant dans les meilleures conditions ${ }^{43}$. Il s'agit d'une succession de charges très classique. Sans doute originaire de Rome même, Victorinus commença sa carrière par les milices équestres qui le menèrent successivement en Bretagne ${ }^{44}$, puis en Pannonie inférieure ${ }^{45}$ et Dacie. Ensuite Victorinus débuta une carrière procuratorienne dont les premiers postes ne sont pas connus avec certitude, en raison des conditions particulières qui nous ont transmis son cursus. Cela l'entraîna en Galatie ${ }^{46}$, puis en Espagne, avant qu'il ne revienne à Rome pour diriger le ludus magnus avec un salaire ducénaire. Victorinus retrouva ensuite l’armée en

39 CIL VI, 1564 (ILS 1452); CIL VI (8,3), 41130 (Rome). Cf. CPE nº 178.

40 G. Camodeca, « Carriera », 1981, cit. supra, p. 47.

41 S.H.A., Vita Didi Iuliani, III, 6. Voir CIL VI, 654. G. Alföldy, Fasti Hispanienses, Wiesbaden, 1969, p. 143 propose de reconnaître sa carrière dans l'inscription CIL VIII, 15869 (Sicca) sans être toujours suivi (voir la prudence de B.-E. Thomasson, Laterculi praesidium I, Göteborg, 1984, c. 30 n. 23).

42 PIR $^{2}$ F 581 ; CPE, n 139 ; la bibliographie de M. Absil, op. cit., p. 177 nº 39 n’est pas satisfaisante : il faut notamment rajouter H. Devijver, PME, F 100 (avec supplément dans le tome IV).

43 Voir supra n.5.

44 Au sujet de la cohorte Bracarum en Bretagne cf. CIL VII, 237 (ILS 3598) ; RIB 649 et voir les notices de H. Devijver, PME A 43 et A 44 avec supplément dans le t. IV, p. 1419. Sur la cohorte de Bracari, qu'il faut peut-être identifier aux Bracaraugustani, voir aussi P. Le Roux, « Les diplômes militaires et l'évolution de l'armée romaine de Claude à Septime Sévère: auxilia, numeri et nationes », in W. Eck et H. Wolff hrsg., Heer und Integrationspolitik : Die römischen militärdiplome als historiche Quelle, Cologne, 1986, p. 347-374.

45 Cf. J. Fitz, Die Verwaltung Pannoniens in der Römerzeit II, Budapest, 1993, p. 803-805, $\mathrm{n}^{\circ} 460$.

46 CPE, p. 326, texte 2 ; (SEG XXVI, 1712) ; S. Michell, Regional Epigraphic Catalogue of Asia Minor II. The Ankara district, B.A.R. I.S., 135, 1982, p. 301-302 n 397, pl. 15 fig. 15, inscription non signalée par M. Absil, op. cit, p. 177. Cf. PME F. 100 suppl. t. IV. Cette première procuratèle connue a longtemps posé problème. La procuratèle du quarantième des Gaules avait aussi été proposée, ainsi que la procuratèle Asturiae et Gallaeciae. J. Fitz, op. cit., p. 804 discute de ces deux dernières hypothèses sans évoquer la Galatie ailleurs que dans le texte de l'inscription, repris de $C P E$, p. 326. Dans un des tableaux de son Supplément aux carrières procuratoriennes équestres, Paris, 1982, p. 61, H.-G. Pflaum reprend la lecture "proc. [Asturiae] et Gall(aeciae)». Plus qu'à un repentir nous voyons là une inattention du grand savant (comparer avec le tableau récapitulatif du même ouvrage p. 117). 
dirigeant successivement les deux flottes prétoriennes de Ravennes puis de Misène selon l'ordre consacré 47 .

L'obtention du poste d'a rationibus consacra sa carrière procuratorienne et lui ouvrit les portes des grandes préfectures, d'abord celle des vigiles plutôt que de l'annone ${ }^{48}$, puis celle d'Égypte entre juillet 159 et septembre $160^{49}$, sans doute une vingtaine d'années après la fin de ses milices équestres. Il dirigea l'Égypte après le long gouvernement de $\mathrm{M}$. Sempronius Liberalis et y précéda C. Volusius Maecianus, qui est attesté en poste en février 161. À cette date, Victorinus était déjà parti pour Rome afin d’y recevoir le commandement des prétoriens. Au moment de son départ, il fut honoré à Alexandrie d'une inscription par l'un de ses anciens subordonnés, un affranchi impérial ${ }^{50}$. Il assura ensuite pendant quelques mois la garde d'Antonin avant de s'occuper de ses deux successeurs.

Les circonstances firent que Victorinus exerça finalement davantage sa fonction loin de Rome qu'en son sein. L'annonce de l'effondrement des armées de Cappadoce et de Syrie face aux Parthes de Vologèse IV bouleversa la vie de la cour impériale. D'amples mouvements de troupes furent décidés, qui convergeaient vers l'Orient. L’empire se préparait à une guerre importante, il fut décidé que Lucius Vérus partirait pour les régions orientales. Victorinus l'accompagna ainsi qu'un certain nombre de sénateurs et les chefs de certains bureaux palatins ${ }^{51}$. Quel rôle Victorinus joua-t-il dans la conduite des combats? Nous ne le savons pas, mais la comparaison de sa carrière avec celle de son collègue explique clairement qu'on l'ait choisi ${ }^{52}$. Nul doute que son expérience des flottes a pu se révéler utile alors que de nombreuses troupes étaient en déplacement et nécessitaient un soutien logistique que seule la marine était à même de fournir. Deux lettres, citées par l'Histoire Auguste, doivent en théorie concerner notre personnage à ce moment là puisqu'elles concernent un préfet du prétoire s'occupant de l'approvisionnement des légions dirigées par Avidius Cassius durant la guerre parthique ${ }^{53}$. On ne peut pas réellement s'appuyer sur ces documents. Ces lettres sont l'œuvre du faussaire et il est en général impossible d’y

47 Cf. M. Reddé, Mare Nostrum, Les infrastructures, le dispositif et l'histoire de la marine romaine sous l'Empire romain, Rome, 1986, p. 674.

48 Nous renvoyons sur ce point à l'article de M. Christol dans ce même numéro. Huelsen retenait la lecture " praef. u[igilum] », mais H.-G. Pflaum (CPE, p. 326) proposa " praef. [ann(onae)] urbi(s) » (suivi en cela par H. Devijver). Dans son étude des préfets de l'annone, H. Pavis d'Escurac considère que L. Volusius Maecianus fut à la tête de cette préfecture de 152 à 159 (La préfecture de l'annone service administratif impérial d'Auguste à Constantin, Rome, 1976, p. 346-347). Cela excluerait évidemment Victorinus des fastes de l'annone (cf. J. Fitz, op. cit., pp. 804-805). Mais l'hypothèse d'une très longue préfecture pour Maecianus se fonde sur sa présence en 152 parmi les patrons d'un collège d'Ostie (CIL XIV, 250 (ILS, 6174)). On ne peut donc pas exclure que sa préfecture ait été plus courte. M. Sablayrolles, Libertinus Miles, Rome, 1996, p. 488 ne retient pas non plus Victorinus parmi les préfets des vigiles.

49 G. Bastianini, « Lista dei prefetti d’Egitto dal 30a al 299p. », ZPE, 17, 1975, p. 294.

50 (OGIS, 707); (IGRR III, 1103); (ILS 8846). En général située à Tyr par les recueils épigraphiques, cette inscription venait bien en fait d'Égypte comme le soupçonnait le commentaire des IGRR; cf. J. et L. Robert, Bull. ép., 1961, nº 96.

51 S.H.A., Vita Marci, VIII, 10.

52 Cf. A.R. Birley, Marcus Aurelius, Londres, 1966, p. 166.

53 S.H.A., Vita Avidii Cassii, V, 4-12. 
déméler ce qui relève de sa fantaisie de ce qui relève de ses connaissances véritables de l'époque de Marc Aurèle. La mention d'annona militaris dans ces lettres est souvent tenue pour un anachronisme, le faussaire projetant sur le deuxième siècle les pratiques de la fin du troisième ${ }^{54}$. Par ailleurs on admet en général que ce fut L. Aurelius Nicomedes qui supervisait le ravitaillement des armées de la guerre parthique. Même si l'on ne peut avoir de certitudes sur la chronologie de sa fonction qui peut aussi être mise en rapport avec l'expeditio Germanica, la charge du ravitaillement des troupes, durant les années 160, semble confiée à d'autres responsables qu'aux préfets du prétoire ${ }^{55}$.

Lorsque Vérus s'approcha du front, il faut penser que Victorinus vint avec lui, mais si Victorinus resta aux côtés de Vérus, il dut la plupart du temps se tenir éloigné de la zone des combats, à Antioche, Daphné et Laodicée. Il faut aussi penser qu'il accompagna Vérus à Éphèse à l'occasion du mariage avec Lucilla. Au retour du conflit il reçut des décorations importantes : trois couronnes, et sans doute quatre lances pures et quatre fanions $^{56}$ : signe probable qu'il joua, d'une manière ou d'une autre, un rôle important dans la guerre. C'est sans doute après le retour triomphal de Vérus à Rome, dans la seconde moitié de 166, qu'il fut honoré des ornements consulaires, mais resta en charge. De nouvelles menaces étant apparues sur les frontières, Victorinus accompagna à nouveau les empereurs, en direction de la frontière danubienne. Le cortège impérial partit de Rome pour Aquilée vers la fin janvier 168. Comme nous l'avons vu, Victorinus trouva la mort dans cette expédition, dans la seconde moitié de 168, sans qu'il soit nécessaire de penser que ce fut au combat, la peste sévissait alors dans ces régions ${ }^{57}$. La mort de Victorinus semble avoir été un des arguments de Lucius Vérus pour convaincre Marc Aurèle de rentrer à Rome : nous pouvons y voir la confirmation des liens importants qui avaient pu se tisser entre le co-empereur et son préfet mais aussi un indice de l’importance du personnage dans la conduite de la guerre.

54 Cf. Zosime II, 33, 4. On verra à propos de ce passage les critiques de J.B. Campbell, The emperor and the Roman army 31 BC - $A D$ 235, Oxford, 1984, p. 114-115. Après A. Nicoletti, «I prefetti del pretorio e la ricossione dell'annona militare ", Labeo, XV, 1969, p. 177-187, F. Carla, «Tu tantum praefecti mihi studium et annonam in necessariis locis praebe : prefettura al pretorio e annona militaris nel III secolo D.C. ", Historia, 56,1, 2007, p. 82-110 est revenu sur le rôle possible des préfets du prétoire dans le ravitaillement des armées mais peut-être sans tenir assez compte du contexte propre à la Vita d'Avidius Cassius. Nous adopterons, au sujet du rôle possible du préfet du prétoire à l'époque de Marc Aurèle, une plus grande prudence. Voir N.J.E. Austin et N.B. Rankov, Exploratio. Military and Political Intelligence in the Roman World from the Second Punic War to the Battle of Adrianople, Londres, 1998, p. 227-228.

55 Sur L. Aurelius Nicomedes : CIL VI, 1598 ; cf. CPE n 163 et F. Bérard, « La carrière de Plotius Grypus et le ravitaillement de l’armée impériale », MEFRA, 96, 1984, pp. 296-297 avec n. 112 et 116 et p. $311, n^{\circ} 4$.

56 Cf. V.A. Maxfield, The military decorations of the roman army, Los Angeles, 1981, p. 206.

57 Cf. J. Fitz, op. cit., p. 805 ; A.R. Birley, Marcus Aurelius² ${ }^{2}$ Londres, 1993, p. 249-255. 


\section{T. Flavius Constans}

Nous ne savons quasiment rien de ce personnage qui était vraisemblablement originaire de Cologne ${ }^{58}$ en Germanie inférieure. De sa carrière nous ne connaissons qu'une procuratèle centenaire, celle de Dacie inférieure en 138. Il est envisageable, nous l'avons vu, de placer sa préfecture du prétoire sur une très courte durée vers 168 , en tant que collègue de Furius Victorinus et peut-être très brièvement de Bassaeus Rufus, mais cela n'est absolument pas certain.

\section{Bassaeus M(arci) f(ilius) Stellatina Rufus}

Bassaeus Rufus 59 était un italien d'origine fort modeste, puisque n’appartenant sans doute pas, au départ, à l'ordre équestre. Sa tribu est celle de Bénévent, où presque tous les Bassei sont identifiés: c'était vraisemblablement sa cité d'origine ${ }^{60}$. Ses origines obscures firent qu'il fut sans doute régulièrement confronté durant sa carrière à la morgue et à la distinction des aristocrates de l'empire, habiles probablement à lui faire sentir son absence de paideia ${ }^{61}$, prolixes incontestablement en anecdotes désobligeantes sur son ascension sociale, son comportement, son enrichissement incontestable. Dion, signalant par ailleurs les qualités du personnage, insistait sur son manque d'éducation, ses origines pauvres et rustiques, rapportant des anecdotes ironiques sur son ascension sociale ou sur ses maladresses à la cour'62. L'Histoire Auguste, qui ne mentionne pas son nom, gardait encore trace, à la fin du IVème siècle des rumeurs malveillantes qui circulaient à son propos ${ }^{63}$. À la lumière de ces documents

\footnotetext{
58 Cf. W. Eck, op. cit. Il nous semble plus simple de penser qu'il était originaire de Cologne pour expliquer la présence de l'inscription en question que de supposer un déplacement du préfet du prétoire dans ces régions pour faire face à un impératif militaire, ainsi que le faisait A.R. Birley, Marcus Aurelius, Londres, 1966, p. 178 qui plaçait Constans après Repentinus. Il expliquait sa présence en Germanie inférieure par le départ de la légion I Minervia pour l’Orient qui aurait affaibli la défense de la région. Nous ne retiendrons pas non plus l'idée de E. Stein selon laquelle Constans serait directement passé de la procuratèle de Belgique et des Germanies au prétoire, idée qui était encore retenue par W. Meyer, L'administration de la province romaine de Belgique, Bruges, 1964, p. 77-78 (cf. H.-G. Pflaum, « Compte-rendu de W. Meyers, L'administration de la province romaine de Belgique », Gnomon, 37, 1965, p. 390 désormais Scripta Varia II, Paris, 1981, p. 78).

59 PIR ${ }^{2}$, B 69 ; CPE n 162 ; B. Dobson, Die Primipilares, Cologne, 1978, p. 254, n 134 . La bibliographie donnée par M. Absil, op. cit., p. 178-179, n 40 n’est pas satisfaisante.

60 Ainsi CIL IX, 1763 ; voir le commentaire de M. Sartre à IGLS XIII, 9082 (Arabie, Bostra) et F. Jacques, Les curateurs de cités dans l'Occident romain de Trajan à Gallien, Paris, 1983, p. 234236.

61 Sur ce point voir P. Brown, Pouvoir et persuasion dans l'antiquité tardive, tr.fr., Paris, 1998, p. 57-102 et en particulier p. 62. On peut comparer la situation de Bassaeus à celle d'Adventus selon Dion Cassius, LXXIX, 14, 2.

62 Dion Cassius, LXXI, 5, 2-3 (Petr. Patr., Exc. Vat., 117), cf. Exc. Val., 302 et 303. Selon ce dernier extrait il aurait été enrôlé «de force » dans l'armée, mais peut-être n’est-ce qu'une mésinterprétation du compilateur. Noter l'usage que Rostovtseff fit de ces anecdotes et de l'origine modeste de Bassaeus Rufus, voulant y voir la confirmation de la thèse majeure de son Histoire économique et sociale de l'empire romain, tr. fr., Paris, 1988 (1957), p. 459 n. 36 . Nous ne saurions le suivre.

63 C'est en effet Bassaeus Rufus qu'il faut identifier au préfet du prétoire de la dernière lettre apocryphe de la Vita Avidii, XIV, 8 ; cf. M. Durry, Les cohortes, cit. supra, p. 159.
} 
on comprend d'autant mieux qu'une de ses remarques ait provoqué le départ d'Hérode Atticus lors du procès de Sirmium. Bassaeus Rufus offre donc, avec quelques autres, l'exemple d'une réussite exceptionnelle basée sur une longue carrière militaire ${ }^{64}$.

Ses débuts modestes expliquent qu'ils aient été peu détaillés sur l'inscription qui nous a conservé son cursus ${ }^{65}$. Elle ne débute en effet qu'avec son primipilat, il est possible de penser que Bassaeus exerça un centurionat dans la garnison de Rome ${ }^{66}$. Il effectua le triple tribunat au sein des vigiles ${ }^{67}$, des cohortes urbaines et enfin prétoriennes. Avec son second primipilat, cela lui permit d'entrer dans la carrière procuratorienne directement à l'échelon ducénaire. Il commença cette partie de sa carrière en tant que procurateur Asturiae et callaeciae, puis dirigea la province du Norique vers 158-16068. Il fut ensuite procurateur des provinces de Belgique et des deux Germanies ${ }^{69}$, puis a rationibus, sans doute vers 165 avant d'être préfet des vigiles. Poste qu'il occupait au printemps $168^{70}$, peu avant de partir pour l'Égypte. Là-bas il remplaça Q. Baienus Blassianus. Les documents égyptiens placent sa préfecture de juillet 168 - semble-t-il - à mars-avril 16971. La mort de Furius Victorinus mit fin à ses fonctions égyptiennes et imposa son retour à Rome. C. Calvisius Statianus commença alors un long gouvernement de l'Égypte, perturbé par les Boukoloi, qui sévissaient déjà en 168 et qui ne prit fin qu'avec l'écroulement du rêve impérial d'Avidius Cassius.

Si l'on en croit la chronologie tirée des documents égyptiens, Bassaeus arriva à Rome après la mort de Lucius Vérus, mais son cursus précise bien qu'il en fut le préfet du prétoire : il avait donc été nommé par les deux empereurs. Il y retrouva la cour et Marc Aurèle qui préparait un nouveau départ vers les frontières du nord de l'Italie. Nul doute que notre personnage fut invité à participer à l'élaboration des prochaines campagnes. Il commandait les prétoriens, mais surtout il avait une expérience fort longue de l'armée, et aussi des régions menacées puisqu'il avait dirigé le Norique quelques années auparavant. Néanmoins c'est dans un tout autre rôle que nous le voyons avec son collègue Macrinius Vindex dans le plus ancien document à évoquer sa préfecture, tous deux interviennent auprès des autorités de Saepinum et de Bovinum pour faire cesser les troubles soulevés par la transhumance ${ }^{72}$. Bassaeus ne tarda pas cependant à s'éloigner de

64 Voir les importantes remarques de S. Demougin, « Appartenir à l’ordre équestre au IIème siècle ", in W. Eck hrsgb., Prosopographie und sozialgeschichte. Studien zur Methodik und Erkenntnismöglichkeit der kaiserlichen Prosopographie. Kolloquium Köln 24-26 Nov.1991, Cologne, 1993, p. 244-246.

65 CIL VI, 1599 + 31828 (ILS 1326) ; CIL VI, 41141 qui énumère son cursus et ses décorations.

66 M. Durry, op. cit., p. 159.

67 Cf. M. Sablayrolles, Libertinus Miles, 224, Rome, 1996, nº 17.

68 CIL III, 5171 ; ILLPRON, 1632 ; E. Schallmayer, Corpus der griechischen und lateinischen Beneficiarier-Inschriften des Römischen Reiches, Stuttgart, 1990, nº 241 (Norique, Celeia)

69 Notice très brève dans W. Meyer, op. cit., p. 78.

70 CIL XIV, 4500 ; Cf. M. Sablayrolles, Libertinus Miles, C.E.F.R. 224, Rome, 1996, p. 488-489 $\mathrm{n}^{\circ} 15$.

71 G. Bastianini, op. cit., p. 297.

72 CIL IX, 2438 = 4916. Voir infra. 
l'Italie et de ses troupeaux, accompagnant Marc Aurèle vers le nord pour lancer une nouvelle offensive qui s’avéra désastreuse. Les guerres firent qu'il dut rester de longues années loin de Rome aux côtés de l'empereur sur les frontières.

Bassaeus Rufus et son collègue Macrinius Vindex furent-ils en général tous les deux présents en permanence avec Marc Aurèle à Carnuntum, quelles responsabilités militaires furent confiées à Bassaeus? Nous l'ignorons. Compte tenu de sa carrière, nous sommes sans doute en droit de penser qu'il fut engagé au combat, comme son collègue malheureux. Après la mort de Vindex, Bassaeus resta aux côtés de l'empereur. Eut-il immédiatement un nouveau collègue ? Si oui, il peut s'agir de Paternus. Il est aussi possible de penser que Rufus fut seul en charge durant quelques années. Nous le retrouvons, nous l'avons vu, à Sirmium lors du procès d'Hérode Atticus, où il prend une part active ${ }^{73}$. Nul doute qu'une telle situation était assez ordinaire, Marc Aurèle s'investissant pleinement dans l'aspect judiciaire de sa fonction ${ }^{74}$. Par ailleurs Dion Cassius, dans une des anecdotes au sujet de notre personnage, nous le montre à nouveau aux côtés de Marc Aurèle, alors que ce dernier s'entretient avec des interlocuteurs non identifiés ${ }^{75}$. Dans cette scène Bassaeus intervient spontanément, et se ridiculise. Nous avons là un écho des rumeurs de cour promptes à s'acharner sur celui qui n'a pas la distinction naturelle de l'aristocrate bien éduqué, mais aussi un témoignage précieux sur la place de Bassaeus auprès du prince. Enfin nous ne pouvons qu'imaginer le rôle crucial de Bassaeus Rufus lors de la crise de 175, à l'annonce de la rébellion d'Avidius Cassius, puis dans la conduite des enquêtes liées à cette crise et lors du voyage en Orient qui signifia à ces provinces la reprise en main de la situation par Marc Aurèle, ainsi que sa clémence.

Bassaeus Rufus rentra à Rome avec Marc Aurèle, qui ne tarda pas à faire de son fils un second Auguste. Un immense triomphe fut célébré le 23 décembre $176^{76}$. On peut penser que c'est à cette occasion que les deux Augustes lui remirent officiellement les décorations gagnées à l'occasion de la victoire contre les Germains et les Sarmates : trois couronnes, quatre lances pures, quatre fanions, distinctions qui correspondaient au niveau d'un gouverneur consulaire ${ }^{77}$. Bassaeus Rufus fut aussi honoré des ornements consulaires, cela ne saurait signifier qu'il quittait sa charge, puisque Victorinus qui les avait aussi reçus est mort en fonction. Enfin sur proposition des deux empereurs, le sénat décida de lui élever, presque certainement de son vivant ${ }^{78}$, trois statues, ainsi qu'il en avait été pour

\footnotetext{
73 Philostrate, Vie des sophistes, II, 1, 11 (561) (éd. Wright, p. 171). Cf. F. Millar, The Emperor in the Roman World ${ }^{2}$, Londres, 1992, pp. 4-7. Sur Hérode Atticus et ses opposants voir W. Ameling, Herodes Atticus I, Hildesheim, Zürich, New York, 1983, p. 136-151, en dernier lieu B. Puech, Orateurs et sophistes grecs dans les inscriptions d'époque impériale, Paris, 2002, p. 204-210 au sujet de Demostratos.

74 Dion Cassius LXXI, 6.

75 Dion Cassius, LXXI, 5, 3 (Exc. Vat. 117 et Exc. Val. 302).

76 S.H.A., Vita Commodi, XII, 5.

77 Cf. V.A. Maxfield, op. cit., p. 206.

78 Cf. V.A. Maxfield, op. cit., p. 108-109.
} 
Vindex à sa mort, l'une en arme sur le forum de Trajan, une autre en civil vraisemblablement dans le temple d'Antonin le Pieux et enfin la dernière en cuirasse, peut-être dans le temple de Mars Ultor. Les honneurs ne mirent pas fin à ses fonctions, et la table de Banasa ${ }^{79}$, où il figure en septième position parmi les signataires, le montre au sein du conseil de l'empereur le 6 juillet 177. Il nous semble tout à fait légitime de penser qu'il y siégeait encore en tant que préfet du prétoire, il était alors associé à Taruttienus Paternus. Par la suite nous perdons sa trace, il dut se retirer ou mourir peu de temps après, en tout état de cause sans doute avant 180, et nous ne savons pas s'il accompagna une dernière fois l'empereur vers les frontières en 178. Ayant passé presque dix ans en fonction, et cela au coeur du règne et durant des années cruciales, Bassaeus Rufus est sans doute le préfet qui marqua le plus le règne. Son passé militaire explique sans doute qu'on l'ait choisi, ainsi que la réussite de sa charge, car son action fut incontestablement appréciée de l'empereur et de ses contemporains.

Si l'ascension sociale de Bassaeus Rufus est parfois décriée par nos sources, elle profita incontestablement à ses descendants et dépendants. C'est sans doute son fils qu'il faut reconnaître dans Bassaeus Astur, gouverneur d'Arabie connu par deux inscriptions de Bostra ${ }^{80}$. Son cognomen peut faire allusion à la procuratèle de son père en Espagne durant laquelle il a pu naître, ce qui situerait sa naissance vers 158, et par conséquent son gouvernement d'Arabie vers la fin du règne de Commode ou le début de celui de Septime Sévère ${ }^{81}$. Une des deux inscriptions de Bostra nous apprend que le gouverneur eut un fils lui aussi appelé Astur ${ }^{82}$. Le dédicant est un centurion de la légion XXII Primigenia, cantonnée habituellement à Mayence, sa présence en Arabie pouvant s'expliquer par ses origines ${ }^{83}$. Un Bassaeus Astur est aussi connu à Bénévent, berceau de la famille ${ }^{84}$. Le gentilice de nos personnages étant rare, il est logique de penser que c'est aussi un membre de la famille qui était présent lors des Ludi saeculares de 204, et peut-être même le fils du gouverneur d'Arabie ${ }^{85}$. Il est donc légitime de penser que les enfants de Bassaeus Rufus avaient fait souche au sein de l'ordre sénatorial. En revanche, nous ne pouvons pas supposer de lien de parenté directe avec le procurateur M. Bassaeus M.f.

\footnotetext{
79 W. Seston et M. Euzennat, « Un dossier ...», 1971, cit. supra, p. 468-490 (AE 1971, n 534); I. A. M., $\mathrm{n}^{\circ} 94$.

80 IGLS XIII, 9081 et 9082 . La première de ces inscriptions a amélioré la lecture de la seconde, connue depuis 1904 au moins. Cf. PIR ${ }^{2}$ B 66 ; H.-G. Pflaum, « Les gouverneurs de la province romaine d'Arabie de 193 à 305 », Syria, 34, 1957, p. 144 n 39 désormais Scripta Varia II, Paris, 1981, p. 249 (datation très basse) ; E. Birley, « M. Bassaeus Astur : a note », ZPE, 37, 1980, p. 1921 ; M. Sartre, Trois études sur l'Arabie romaine et byzantine, col. Latomus 178, 1982, p. 95 ; B.E. Thomasson, Laterculi praesidium I, Göteborg, 1984, c. 335 n. 43 (prudent sur la datation).

81 E. Birley, op. cit.

82 (AE 1920, nº 73) ; IGLS XIII, 9082.

83 Voir le commentaire à IGLS XIII, 9082. La question se pose des rapports éventuels de l'un ou l'autre Astur à la Germanie.

84 CIL IX, 1763.

85 CIL VI, 32334. Cf. E. Birley, op. cit.
} 
Palatina Axius ${ }^{86}$ car la tribu de ce dernier n'est pas la même que celle de Bassaeus Rufus, mais nous pouvons penser avec E. Birley qu'il s'agissait d'un fils d'un affranchi du préfet du prétoire 87 et qu'il put bénéficier de sa protection au moins au début de sa carrière.

\section{Macrinius [Claudia] Vindex}

Nous savons bien moins de choses sur Macrinius Vindex ${ }^{88}$ que sur son collègue, son temps de fonction a été plus court et le hasard a voulu que nous ne retrouvions aucune des trois bases de statues qui lui furent érigées à Rome vers 172, sans doute à proximité des endroits où furent placées en 175 les statues de Bassaeus Rufus. Nous connaissons en revanche bien mieux la vie et la carrière de son fils $M$. Macrinius Avitus M(arci) f(ilius) Claudia Catonius Vindex ${ }^{89}$. La famille fut longtemps tenue pour italienne, mais l'inscription dans la tribu Claudia ainsi que la formation du gentilice Macrinius et le cognomen Vindex ont plus récemment fait chercher les origines de cette famille dans les provinces occidentales de l'empire. Plus précisément les hypothèses se sont portées sur Camulodunum, l'actuelle Colchester en Bretagne, et la colonia Agrippinensis, Cologne en Germanie inférieure $^{90}$. On ne saurait actuellement avoir de certitude absolue. Enfin, le nom du fils suppose vraisemblablement que le père, le préfet du prétoire, épousa une Catonia qui appartenait peut-être à l'ordre sénatorial ${ }^{91}$.

De la carrière de Vindex père avant sa préfecture nous ne connaissons qu'un poste. Il fut procurateur gouverneur de Dacie Porolissensis en $154^{92}$, poste qui était alors de rang ducénaire. En moins de quinze ans Vindex parcourut les dernières étapes du cursus équestre pour arriver au sommet, il faut sans doute lui supposer d'autres postes ducénaires (les flottes prétoriennes après Victorinus ?), vraisemblablement au moins un poste dans les bureaux palatins. Nous sommes sûrs qu'il ne fut pas préfet d'Égypte, car tous semblent connus ${ }^{93}$. Il faut enfin envisager les deux grandes préfectures romaines. S'il fut préfet des vigiles ce ne peut-être que très brièvement, puisque son collègue Bassaeus Rufus, nommé sensiblement à la même époque à la tête du prétoire le fut jusqu'en 168 et qu'il semble difficile de placer Vindex entre Rufus et Umbricius Aemilianus, dont la préfecture à la tête des vigiles se place vers 161-166 ${ }^{94}$. Quant aux fastes de l'annone, ils

86 CIL X, 1795 (ILS 1401) (inscription transportée à Naples, peut-être en provenance de Pouzzoles ou de Bénévent) ; cf. PIR ${ }^{2}$ B 68 ; CPE n 207 ; PME B 16 avec suppl. t. IV ; F. Jacques, Les curateurs, cit. supra, p. 234-236.

87 E. Birley, op.cit., p. 19-21.

88 PIR $^{2}$ M 25 ; CPE, n ${ }^{\circ} 161$; M. Absil, op. cit., p. 180 n 40 n’est pas satisfaisant : l’inscription de Saepinum n'est pas signalée et semble même ignorée si l'on en croit la note 20.

89 CIL VI, 1449 et p. 3805 (ILS 1107). Cf. PIR ${ }^{2}$ M 22 ; CPE, n 188 ; PME M 4 ; J. Fitz, op. cit., $\mathrm{n}^{\circ}$ 504, p. 834-836.

90 Cf. A.R. Birley, E.O.S. II (Tituli 5), Rome, 1982, p. 535 ; F. Jacques, op. cit., p. 48 n. 3.

91 Voir les observations de O. Salomies à ( $A E$ 2000, $\left.n^{\circ} 1138\right)$.

92 CIL XVI, 110 ; R.M.D., 47.

93 Cf. G. Bastianini, op. cit., p. 292-297.

94 CIL XIV, 4509 ; cf. M. Sablayrolles, Libertinus Miles, Rome, 1996, p. 488 n 14. 
sont occupés jusqu'en 159 par Volusius Maecianus ${ }^{95}$, et nous connaissons sous Marc Aurèle et Lucius Vérus les préfectures d'Ulpius Saturninus ${ }^{96}$ et de Baienus Blassianus ${ }^{97}$. Il est donc aussi envisageable que Vindex ait été à la tête de l'annone en 167, mais seulement de manière assez brève.

Nous ne connaissons pas le moment précis où Vindex fut appelé par l'empereur pour prendre la tête du prétoire. Cependant, puisque son nom apparaît après celui de Bassaeus Rufus dans l'inscription de Saepinum, il faut penser que Vindex avait moins d'ancienneté que Bassaeus Rufus dans le poste. Comme le dossier de Saepinum relate des échanges épistolaires qui ne peuvent pas s'être déroulés trop longtemps après 168, date où nous sommes sûrs que Cosmus était en charge ${ }^{98}$, il faut penser que Vindex prit sans doute ses fonctions vers le début de 169. Nous pouvons envisager qu'il succédait à Flavius Constans.

Il accompagna lui aussi Marc Aurèle vers les frontières des régions Danubiennes. Là il assuma un commandement important. Il prit une part active aux combats contre les Marcomans dans lesquels il trouva finalement la mort, au plus tard dans la première moitié de $172^{99}$. Trois statues furent érigées en son honneur. La mort de Vindex fut assurément un rude coup porté aux forces romaines au moment où elles venaient de rétablir leur situation après les désastres de l'année 170. Bien qu'ignorant l'essentiel de son cursus nous pouvons sans doute penser que Macrinius avait développé et révélé dans ses différents postes des compétences militaires. Nul doute en tout cas que ce sont de telles compétences que Marc Aurèle attendait de ces préfets au début des années 170 quand il leur confia le commandement d'armées importantes ${ }^{100}$.

Si Vindex était tombé au combat, son fils continuait à contribuer brillamment à la défense de l'empire. Il s’y était déjà illustré du vivant de son père, mais ne poursuivit pas une carrière équestre ayant bénéficié vers 170 d'une adlection parmi les clarissimes de rang prétorien. Il mourut à 42 ans gouverneur de rang consulaire en Mésie inférieure. Il avait épousé Iunia Flaccinilla $^{101}$ qui lui avait donné au moins une fille, Macrinia Rufina. Macrinius s'était allié à une très puissante famille. Les Iunii Rufini avaient notamment eu deux consuls ordinaires en 153 et 155, et comptaient sans doute des liens avec bien d'autres puissants personnages ${ }^{102}$. La famille de

\footnotetext{
95 Cf. H. Pavis d'Escurac, La préfecture de l'annone service administratif impérial d'Auguste à Constantin, Rome, 1976, p. 346-347.

96 CIL II, 1180 (ILS 1403). Cf. H. Pavis d’Escurac, op. cit., p. 348. Il s'agit de la carrière de Sex. Iulius Possessor dont les fonctions annonaires ont été interprétées de diverses manières. Voir notamment H.-G. Pflaum, CPE, 185 avec Suppl., 1982, p. 50-51

97 H.-G. Pflaum, Suppl., 1982, nº 126, p. 41-42.

98 Cf. CIL VI, 455 ; ( $A E$ 1995, n 59=91) datée de la fin septembre 168.

99 Dion Cassius, LXXI, 3,5 (Xiphilin, 259, 26 - 260, 6). Vindex mourut avant que Marc Aurèle ne prenne le titre de Germanicus, le 15 octobre 172 (S.H.A., Vita Commodi, XI, 14). Voir aussi S.H.A., Vita Marci, XXII, 2.

100 S.H.A., Vita Marci, XXII, 2.

101 PIR $^{2}$, I 860 ; FOS, 471.

102 A. Iunius Rufinus fut consul en 153 et proconsul d'Asie en 169-170 et son frère M. Iunius Rufinus Sabinianus fut consul en 155 et proconsul d’Afrique en 172-173 : voir W. Eck, «A. Iunius Rufinus, proconsul Asiae unter Marc Aurel, und seine familie », dans P. Scherrer, H. Taeuber,
} 
Macrinius eut par la suite une descendance puisque nous connaissons un M. Claudius Macrinius Vindex Hermogianus qui fit une carrière sénatoriale au début du troisième siècle ${ }^{103}$.

\section{P. Taruttienus P(ublii) f(ilius) Poblilia Paternus}

Le gentilice exact de Taruttienus Paternus ${ }^{104}$ avait été déformé par les sources littéraires ${ }^{105}$, il n'est connu avec certitude que depuis la publication de la table de Banasa, et a été confirmé par une inscription de Cilicie ${ }^{106}$. Ce gentilice ne se trouvant qu'à Rome ${ }^{107}$, il faut peut-être y situer les origines de notre personnage, plutôt qu'à Vérone ${ }^{108}$. Nous ignorons tout de ses débuts et ne le découvrons, grâce à Dion Cassius, que lorsqu'il est $a b$ epistulis latinis de Marc Aurèle vers 170-17109. En fait de correspondance, c'est de diplomatie militaire dont s'occupe alors Paternus. Il est en effet reçu par les Cotins que Marc espérait gagner à l'alliance romaine contre les Marcomans. Mais ce petit peuple aux lointaines origines celtes, et aux voisins puissants, déçut les espérances de l'empereur. Ils refusèrent l'alliance et maltraitèrent Paternus. Ce passage est extrêmement intéressant par ce qu'il nous montre de l'activité de l'ab epistulis latinis au moment des guerres du règne de Marc Aurèle. Dans ce poste Paternus avait sans doute succédé à C. Calvisius Statianus ${ }^{110}$. Ce dernier étant préfet d’Égypte dès

H. Thür (éd.), Steine und Wege. Festschrift für Dieter Knibbe zum 65 Geburtstag, Vienne, 1999, pp. 299-302 et les notices de la $P I R^{2}$ de Q. Pomponius Maternus (P 736) et Pomponia Triaria (PIR ${ }^{2} \mathrm{P}$ 780), fille du consul de 153 qui épousa Erucius Clarus $\left(P I R^{2}, \mathrm{E} 95\right)$.

103 CIL X, 4860. Cf. PIR ${ }^{2}$, C 918 ; voir aussi CIL X, 4861.

104 PIR T 24 ; CPE, n 172 ; M. Absil, op. cit., p. 182-183 n 43 n’est pas satisfaisant. Il omet notamment de signaler M. Christol et S. Demougin, « Notes de prosopographie équestre VI : Un chevalier originaire de Cilicie », ZPE, 74, 1988, p. 14-21, voir (AE 1988, n 1049).

105 Dion Cassius, LXXII, 5 donnait «Tarrutenius » et en LXXI, 12 « Tarrunius », l'Histoire Auguste donnait aussi Tarrutenius, en revanche les sources juridiques donnaient Tarruntenus. Sur ce point voir M. Christol, « Remarques sur la carrière de L(ucius) Mummius Faustianus, consul ordinaire en 262 », dans A. Akerraz, P. Ruggeri, A. Siraj et C. Vismara dir., L'Africa romana XVI, Rabat 2004, Rome, 2006, p. 1851 n. 46-47.

106 (SEG XXVIII, 1255) ; (J. et L. Robert, Bull. ép. 1979, 596) classée par inadvertance à Anazarbe ; G. Dagron et D. Feissel, Inscriptions de Cilicie, Paris, 1987, n 87 attribuée à Mopsueste ; cf. M. Christol et S. Demougin, op. cit., p. 16-17.

107 CIL VI, 27118 concernant sans doute notre personnage et relu ainsi par CIL VI, 41274 ; cf. M. Christol et S. Demougin, op. cit., p. 16.

108 M. Absil, op. cit., p. 28. C'est sur la base des anciennes lecture de son gentilice (Tarrutenius) que l'on plaçait ses origines en Italie du Nord, ainsi H.-G. Pflaum et E. Marec, « Deux carrières équestres d'Hippone », Libyca, 1, 1953, p. 214, désormais Scripta Varia I, Paris, 1978, p. 40 qui évoquaient Turin sur la base de CIL III, 3565.

109 Dion Cassius, LXXI, 12, 3 (Exc. de legationibus gentium, 58). La date est donnée par la mention du gouvernement de Cornelius Clemens en Dacie; sur ce dernier voir I. Piso, Fasti Provinciae Daciae I, Bonn, 1993, p. 103-105. Cf. A.R. Birley, Marcus Aurelius, Londres, 1966, p. 233 ; M. Stahl, «Zwischen Abgrenzung und Integration : Die Verträge der Kaiser Mark Aurel und Commodus mit den Völkern jenseits der Donau », Chiron, 19, 1989, p. 289-317 et notamment p. 302. Nous ne comprenons pas pourquoi M. Durry, Les cohortes, cit. supra, p. 161 précise qu'il fut $a b$ epistulis «jusqu'en 174 » et pas plus pourquoi N.J.E. Austin et N.B. Rankov, Exploratio. Military and Political Intelligence in the Roman World from the Second Punic War to the Battle of Adrianople, Londres, 1998, p. 140 situent l'épisode des Cotins en 173.

110 CIL V, 3336 (ILS 1453) ; cf. CPE nº 166. 
février $170^{111}$, il est possible que Paternus ait pris ses fonctions un peu auparavant. Calvisius Statianus avait lui même succédé à T. Varius Clemens vers 166. Si nous ignorons le reste de la carrière de Statianus nous connaissons très bien celle de Clemens ${ }^{112}$. Clemens qui présentait d'incontestables aptitudes militaires avait été vraisemblablement nommé vers 162, au début de la guerre parthique. Il remplaçait Sex. Caecilius Crescens Volusianus dont le cursus avait été bien différent et surtout bien moins militaire ${ }^{113}$. Il est possible de penser que les guerres entraînèrent un infléchissement de la fonction, mettant l'accent sur la correspondance militaire. Il ne faut pas cependant exagérer ce phénomène, mais il nous semble légitime de supposer que Paternus avait déjà révélé des aptitudes militaires lorsqu'il arriva à ce poste. En revanche, on ne saurait supposer que ce sont uniquement des compétences martiales qui motivèrent son choix: Paternus, nous le verrons, fut aussi un juriste reconnu. Ses compétences littéraires et administratives comptèrent sans doute fortement. Terminons en ajoutant que le dernier $a b$ epistulis latinis connu pour le règne de Marc Aurèle ne présente pas un cursus spécialement militaire ${ }^{114}$. Malgré son échec chez les Cotins, Paternus poursuivit sa carrière.

C'est parmi les signataires de la table de Banasa que nous le retrouvons, le 6 juillet 177, à une place que nous ne pouvons comprendre, nous l'avons vu, que comme celle de préfet du prétoire, collègue de Bassaeus Rufus. Nous ne savons pas à quelle date placer son entrée en fonction. Après sa fonction d'ab epistulis, il a pu exercer un poste intermédiaire ${ }^{115}$. Mais il n'est pas impossible de penser qu'il ait directement succédé à Macrinius Vindex ${ }^{116}$. Dans ce cas la durée de ses fonctions à la tête des prétoriens serait bien plus longue que ce que l'on suppose habituellement. Il serait alors directement passé du bureau de la correspondance à la préfecture. Cet avancement rapide, qui fait fi des autres grandes préfectures, n’est pas inenvisageable et pourrait par ailleurs tout à fait s'expliquer par le contexte particulier de la mort de Vindex. Son préfet étant mort au combat, Marc Aurèle aurait cherché son remplaçant au plus près. Au demeurant Paternus avait prouvé, peu de temps auparavant, ses qualités et la confiance que l'on pouvait avoir en lui. Dans ce cas Paternus serait passé devant Calvisius Statianus qui resta en Égypte jusqu'en 175, date à laquelle il prit fait et cause pour l'usurpation d'Avidius Cassius. Statianus avait incontestablement l'ancienneté requise pour être préfet du prétoire lors de la mort de Vindex, mais il était alors à la tête d'une province troublée et remuante et était même en partie débordé par la situation. Il n'est pas non plus impossible de penser que Macrinius Vindex ne fut pas immédiatement remplacé, Bassaeus Rufus restant seul en poste durant une période assez longue. Diverses circonstances ayant pu par la suite motiver le retour à un

\footnotetext{
111 Cf. G. Bastianini, op. cit., p. 298.

112 H.-G. Pflaum, Suppl., n 156 ; J. Fitz, op. cit., n 473, p. 813-814.

113 CIL VIII, 1174 (ILS 1451); cf. CPE, nº 142.

114 CIL VI, 1564 (ILS 1452) ; CIL VI, 41130 ; cf. CPE n 178.

115 M. Christol, « Remarques sur la carrière ...», 2006, cit. supra, p. 1852.

116 Chronologie prudemment suggérée par M. Christol et S. Demougin, “Notes de prosopographie équestre VI : Un chevalier originaire de Cilicie”, ZPE, 74, 1988, p. 17.
} 
couple de préfets, et notamment le retour à un couple d'Augustes à la fin de l'année 176, lorsque Commode reçut le titre.

La seconde expédition germanique fut, pour Paternus, l'occasion de se confronter à nouveau aux barbares. Durant ces campagnes, Marc Aurèle lui confia le commandement d'une force de grande taille et l'envoya sur le théâtre d'opérations - dont la localisation exacte nous est inconnue. La bataille dura toute la journée mais se termina par une nette victoire romaine, Marc fut salué imperator pour la dixième fois, ce qui place l'épisode en 179117. Les compétences militaires de Paternus étaient donc pleinement reconnues par ses contemporains, et confirmées par les événements. Mais il y a plus, nous sommes assurés de l'intérêt de Paternus pour l'armée, puisqu'il y consacra une oeuvre notable, plusieurs volumes De re militari ${ }^{118}$ dont le souvenir et de maigres épaves nous ont été transmis en particulier par les importantes compilations juridiques de la fin de l'antiquité. Car, dans ses livres, c'est en juriste que Paternus s’intéressa à l'armée. Il faut donc compter, à côté de ses compétences militaires, un domaine de compétence juridique. On ne doit pas l'exagérer. Paternus était loin d'être l'égal des grands juristes de l'époque antonine, sa place et ses compétences étaient infiniment plus modestes que celle de personnages comme Salvius Iulianus, Volusius Maecianus ou Cervidius Scaevola119. Il n'en reste pas moins que ses compétences dans le domaine du droit étaient sérieuses et solides et que leur application au domaine militaire fut reconnue et fit même date. Paternus semble avoir été le premier juriste à rédiger un traité De re militari. Dans les faits, pour ce que l'on peut en juger d'après les fragments transmis, Paternus semble s'être limité à l'aspect pénal du droit militaire. Considérant la disciplina dans son aspect le plus répressif et traditionnel - d'où l'importance attachée au sacramentum militiae -, il codifia une situation existant depuis longtemps, s'attachant ainsi au fonctionnement de l'administration militaire, à sa règlementation. Il est, pour l'empire, le premier exemple connu qui élabore véritablement une synthèse des règlements internes à l'armée et de leurs conséquences pénales. Ce faisant Paternus restreignait le domaine d'application de la disciplina à un cadre bien déterminé, proche de notre sens actuel du mot discipline ${ }^{120}$. Son travail inaugurait ainsi une série d'importants ouvrages puisque l'on retrouve des préoccupations semblables chez Arrius Menander et que Paternus était cité dans le De re militari de Macer. La postérité du travail de Paternus fut donc

117 Dion Cassius, LXXI, 33, 3-4 (Xiphilin, 267).

118 M. Durry, Les cohortes, cit. supra, p. 161 parle de quatre livres, chiffre précis dont nous n’avons pas retrouvé l'origine, peut-être était-ce une confusion avec les quatre livres de l'ouvrage homonyme d'Arrius Menander?

119 Sur la place des grands juristes autour du prince à cette époque voir M. Christol, « Le conseil impérial, rouage de la monarchie administrative sous les Antonins et les Sévères », dans R. Haensch et J. Heinrichs dir., Herrschen und Verwalten. Der Alltag der römischen Administration in der Hohen Kaiserzeit, Cologne, 2007, p. 31-59.

120 Nous empruntons l'essentiel de ce développement à l'important ouvrage de J. VendrandVoyer, Normes civiques et métiers militaire à Rome sous le principat, Clermont-Ferrand, 1983, p. 317-321. 
remarquable. Végèce s'y réfère dans l'introduction de son ouvrage ${ }^{121}$. Le Digeste le cite deux fois directement ${ }^{122}$, et une fois à travers l'œuvre de Macer $^{123}$. Nul doute que ce travail de codification et de rationalisation correspondait à un intérêt particulier de Paternus. Il s'inscrit cependant particulièrement bien dans le climat général du règne. Les guerres longues et les déplacements de la cour sur les frontières mettaient en effet en contact prolongé avec l'armée des personnages tels que Paternus. Plus généralement, des milieux cultivés assez divers se penchèrent sur la chose militaire. Même si sa date reste controversée, il nous semble que le $D e$ munitionibus castrorum du Pseudo-Hygin peut correspondre à ce contexte particulier. Ne nous le cachons pas, des raisons courtisanes motivaient cet intérêt pour les affaires martiales et les résultats furent sans doute souvent moins brillants que l'oeuvre de Paternus, comme en atteste la compilation hative de stratagèmes par Polyen. Pour en revenir à Paternus, il faut noter que la place accordée à la disciplina rejoint, par le droit, les recommandations et les observations du rhéteur Fronton lors de la guerre parthique $^{124}$. On peut penser aussi que les difficultés des combats et la nécessité de recruter des soldats qui n’étaient pas nécessairement volontaires posaient fortement la question de la discipline. Par ailleurs, la codification du droit pénal correspond à l'intérêt que Marc Aurèle portait à la pratique juridique et aux procès, $\mathrm{y}$ compris en période de guerre et au coeur de provinces frontières. Enfin cette importance accordée au judiciaire peut aussi être mise en rapport avec la fonction de préfet du prétoire elle-même. Homme de plume tout autant que d'épée, Paternus «unissait la connaissance des choses de l'armée à celle du droit »125, dès lors il est possible de le considérer comme un précurseur des préfets du prétoire juristes de la dynastie des Sévères, mais aussi de le placer dans la continuité de Cornelius Repentinus qui certes n'avait pas de compétences militaires, mais maîtrisait sans doute bien les lettres et le droit. Le personnage et la carrière de Paternus apparaissent donc comme la synthèse de compétences qui relevaient en général d'individus aux spécialisations différentes mais qui n'en étaient pas moins toujours associées au coeur de l'imaginaire politique romain dans leur complémentarité et dans leur alternance: afin que les périodes de guerres et de paix soient bien gouvernées, il était nécessaire que la majesté impériale soit décorée par les armes et soutenue par les lois comme le rappelait encore, au VIème siècle, la préface aux Institutes.

121 Végèce, Epitome rei militaris, I, 8 : « ...quae Paternus diligentissimus iuris militaris adsertor in libros redegit ».

122 D’une part, Digeste XLIX, 16, 7 qui cite son livre II au sujet des traîtres qui doivent être torturés et tués après avoir été démis. Ce passage doit être envisagé dans le cadre de la répression de la désertion. Voir P. Cosme, "Le châtiment des déserteurs dans l'armée romaine ", Revue historique de droit français et étranger, 81 (3), 2003, pp. 287-307 et notamment p. 291. D'autre part Digeste L, 6, 7 qui cite son livre I. Il s'agit du plus long passage conservé, il définit les immunes et en donne une longue liste. Voir en dernier lieu sur ce passage J. Nelis-Clément, Les beneficiarii : militaires et administrateurs au service de l'empire (Ier s. a. C. - VIe s. p. C.), Bordeaux, 2000, p. 64.

123 Digeste XLIX, 16, 12, 1 ; M. Absil, op. cit., p. 182 indique à tort XLIX, 16, 2, 1. Le passage concerne l'octroi des congés par les chefs militaires, congés qui doivent être donnés avec parcimonie, un passage d'un règlement disciplinaire d'Auguste étant cité à l'appui.

124 Cf. Fronton, Principia Historiae, 11-13 et Ad Verum II, 19.

125 M. Durry, Les cohortes, cit. supra, p. 161. 
Mais ses grandes qualités n'empêchèrent pas Paternus de connaître une fin de carrière malheureuse. La chute de Paternus fut en effet une conséquence de la première grande crise politique du règne de Commode, la conjuration de Lucilla126. Pourtant Paternus, comme les autres proches de Marc Aurèle, avaient sans doute assuré la transition d'un règne à l'autre sans problème immédiat. Et Paternus était rentré à Rome aux côtés de Commode, un fois les opérations militaires achevées, en octobre $180^{127}$. Le rôle exact de Paternus dans le complot qui eut lieu environ un an plus tard nous échappe. Dion précise que Paternus fut éliminé avec Salvius Iulianus et Vitrasia Faustina ${ }^{128}$. Dans ce passage l'historien bithynien s'attache à défendre la mémoire du préfet qui selon lui était innocent : s’il avait voulu tuer Commode, il l'aurait pu aisément alors qu'il commandait les prétoriens, mais ne l'avait pas fait. Au contraire la biographie de Commode dans l'Histoire Auguste érige Paternus en acteur direct de la conspiration. Il l'aurait appuyée, puis ayant constaté son échec, il tenta d'en atténuer la répression et organisa la suppression du favori de Commode, Saotérus ${ }^{129}$. De nombreuses personnalités furent victimes de la répression aux côtés de Paternus ${ }^{130}$. Qu'il ait participé ou non au complot, Dion ${ }^{131}$ et l'Histoire Auguste s'accordent lorsqu'ils attribuent un rôle essentiel à Tigidius Pérennis dans son élimination. De même ces deux sources indiquent qu'au moment de son procès et de son élimination, Paternus avait été privé de la direction du prétoire par l'effet d'une adlectio inter consulares ${ }^{132}$. Il fut ainsi habilement éloigné des prétoriens qui auraient pu éventuellement réagir à son élimination ${ }^{133}$. Enfin il faut remarquer que sa fin tragique n’entraîna pas de damnatio memoriae pour Paternus : à la différence de celui de Pérennis son nom n'est pas effacé sur la table de Banasa. Nous possédons vraisemblablement des fragments de deux inscriptions romaines dont l'une portait son cursus ${ }^{134}$ et l'autre son épitaphe ${ }^{135}$.

Les circonstances de la mort de Paternus ont au moins le mérite de nous permettre de cerner la place qu'il occupait au sein des élites dirigeantes de l'empire au sommet de sa carrière. Nous constatons à nouveau combien il était facile pour les enfants d'un préfet de pénétrer dans les cercles les plus huppés des clarissimes. La fille de Taruttienus était en effet promise au fils

\footnotetext{
126 En dernier lieu voir A. Molinier-Arbo, « À qui profitait la conjuration de Lucilla ? réflexions sur un passage des Caesares de Julien », L’Antiquité Classique, 76, 2007, p. 119-132.

127 S.H.A., Vita Commodi, XII, 7.

128 Dion Cassius, LXXII, 5, 2 (Exc. Val., 314). Sur Vitrasia Faustina - dont Dion ne précise pas le nom - voir S.H.A., Vita Commodi, IV, 10..

129 Cf. H.-G. Pflaum, « La valeur de l'information historique », 1972, cit. supra, p. 203.

130 S.H.A., Vita Commodi, IV.

131 Dion Cassius, LXXII, 10 (Xiphilin, 273).

132 Dion Cassius, LXXII, 5, 1 et S.H.A., Vita Commodi, IV, 7. Voir A. Chastagnol, « L’Histoire Auguste et le rang des préfets du prétoire ", dans Recherches sur l'Histoire Auguste, Bonn, 1970, p. 62 ; Id., Le sénat romain à l'époque impériale, Paris, 1992, p. 106, 132, 139; M. Christol, « Remarques sur la carrière ... », 2006, cit. supra, p. 1852.

133 Cf. M. Durry, op. cit., p. 180 et 184. M. Absil, op. cit., p. 74 et 80 parle des ornements consulaires et non d'adlectio. Seule l'adlectio explique que Paternus ait dû quitter le prétoire.

134 CIL VI, 41273 qui devait mentionner les ornements consulaires et des décorations.

135 CIL VI, 41274 (relecture de CIL VI, 27118).
} 
de Salvius Iulianus ${ }^{136}$, lui-même fils du célèbre juriste et consul ordinaire de 175 , et un des personnages clé de la réussite de la prise de pouvoir par Commode à la mort de Marc. Nous constatons aussi que Taruttienus avait sans doute pu avoir une influence sur un certain nombre de nominations à des postes importants de l'administration impériale: son ami Vitruvius Secundus, qui partagea sa chute, avait été $a b$ epistulis ${ }^{137}$. On peut penser que Paternus durant sa carrière avait déployé un réseau de relations considérables, et qu'il avait assuré un nombre important de recommandations, favorisant les carrières de nombreuses personnes. Une inscription de Mopsueste en Cilicie en témoigne clairement ${ }^{138}$. Il s'agit d'une dédicace faite par un tribun de la légion IV Scythica qui auparavant se nommait Demetrios fils de Demetrios. Il s'agit à l'évidence d'un notable local important : l'obtention de la citoyenneté romaine lui permit d'entrer dans l'ordre équestre et d'exercer une milice dans la légion de Zeugma. Ce changement de statut personnel modifia bien sûr son onomastique, et il prit le nom de L. Aurelius Taruttienus Demetrios. Il reçut donc sa citoyenneté de Commode, dans les premiers mois du règne, avec la recommandation du préfet du prétoire ${ }^{139}$. Notons que rien n'oblige à penser que Demetrios ait nécessairement accompli sa milice avant la mort de son protecteur. Enfin nous pouvons compter, même si nous ignorons les liens exacts qu'ils eurent avec Paternus, un grand nombre des dernières victimes de la répression de la conjuration de Lucilla et de ses suites comme étant fort probablement liées à notre personnage. Nous retrouvons là des personnages très prestigieux et qui avaient été très proches de Marc Aurèle, comme les Quintilii140. Paternus eut une postérité familiale qui survécut à sa déchéance. Elle apparaît notamment dans une inscription de Dougga datant des années 270 qui mentionne un clarissime nommé L. Mummius Faustianus Tarruntenius Paternus, fils d'une Tarruntenia Paulina et du consul ordinaire de $262^{141}$.

\section{Tigidius Perennis}

Les fonctions de Perennis apparaissent toujours, dans nos sources, en association avec le règne de Commode ${ }^{142}$. L’Histoire Auguste ne précise pas

136 S.H.A., Vita Commodi, IV, 9.

137 A. Daguet, «Vitruvius Secundus, ab epistulis : un lettré africain ?», Revue des Études Augustiniennes, 34, 1988, p. 3-31.

138 (SEG 28, 1255) (cf. J. et L. Robert, Bull. ép. 1979, n 596); G. Dagron et D. Feissel, Inscriptions de Cilicie, Paris, 1987, $\mathrm{n}^{\circ}$ 87. Voir PME t. IV, A 257bis et surtout M. Christol et S. Demougin, « Notes de prosopographie équestre VI : Un chevalier originaire de Cilicie », ZPE, 74, 1988, p. 14-21 (AE 1988, $n^{\circ}$ 1049). Sur les autres officiers de la IV Scythica, très mal connus pour notre époque : H. Devijver, « Commanders and officers of Legio IIII Scythica », in D. Kennedy et al., The Twin Towns of Zeugma on the Euphrates. Rescue Work and Historical Studies, J.R.A. Suppl. 27, Portsmouth, 1998, p. 205-232 et notamment p. 214

139 M. Christol et S. Demougin, op. cit., p. 18-20.

140 PIR2 Q 21, 22, 24, 27.

141 (AE 1998, $n^{\circ}$ 1569) (Afrique proconsulaire, Thugga). Voir M. Christol, « Remarques sur la carrière ...», 2006, cit. supra, p. 1839-1870 et plus particulièrement 1851-1854.

142 Cf. G. Bersanetti, "Perenne e Commodo », Athenaeum, 29, 1951, XXIX, p. 151-170 ; H.G. Pflaum, «La valeur de l'information historique », 1972, cit. supra, p. 205 et Suppl., 1982, p. 48 ; M. Absil, op. cit., p. 184-185. 
à quel moment il débuta sa charge ${ }^{143}$. Elle l'associe cependant étroitement à Paternus dans un passage qui laisse entendre que Perennis ne fut pas choisi par Commode ${ }^{144}$. Le récit de Dion Cassius, malheureusement abrégé, insiste sur son rôle à la tête du prétoire à la suite de Paternus ${ }^{145}$, mais atteste aussi qu'ils furent collègues ${ }^{146}$. L'Histoire Ecclésiastique d'Eusèbe ne fait que citer son nom sans préciser sa fonction, alors qu'il dirigeait le procès du chrétien Apollonius ${ }^{147}$. Les Actes du procès ${ }^{148}$ n'éclairent pas la fonction de notre personnage et se révèlent parfois erronés ${ }^{149}$. Seul Hérodien nous en apprend un peu plus : selon lui, c'est Commode qui plaça Perennis à la tête des prétoriens ${ }^{150}$. Plus intéressant encore, il précise que Perennis était italien d'origine et qu'il passait pour avoir des qualités militaires, raison pour laquelle il avait été désigné comme préfet du prétoire. Nous retrouvons là l'importance des préoccupations militaires, et des profils de carrière correspondants, que nous avons constatée pour nos autres préfets. Il faut remarquer aussi que, comme Paternus, Perennis ne gouverna pas l’Égypte. Si Commode a véritablement choisi Perennis, peu après la mort de Marc, il ne s'est guère alors écarté des choix qu'avait faits son père. Cela semble encore plus évident lorsque l'on songe à la place éminente qu'occupait déjà Perennis en juillet 177 ainsi que l'a montré la table de Banasa ${ }^{151}$. Dès lors il nous semble légitime de penser que Perennis a été placé à la tête du prétoire par Marc Aurèle lui-même, à l'extrême fin de son règne, pour remplacer Bassaeus Rufus aux côtés de Paternus ${ }^{152}$. Le discrédit attaché durablement à son nom après sa chute peut expliquer que l'historiographie ne l'associa qu'à Commode, lui-même passablement discrédité après sa mort. Dès lors les historiens du règne purent faire le récit de l'entente curieuse d'un souverain maniaque et paresseux avec un arriviste sournois et sanguinaire, tous deux unis contre le sénat. On ne saurait se satisfaire de cette caricature qu'Hérodien comme l'Histoire Auguste se plaisent à construire. Dion Cassius présente en effet une autre image de Perennis. S’il reconnaît le rôle incontestable du personnage dans la chute de Paternus, il insiste sur la

143 S.H.A., Vita Commodi, IV, 7, V-VI, 2 ; Vita Pertinacis, III, 3.

144 S.H.A., Vita Commodi, XIV, 8 : « Praefectos Paternum et Perennem non diu tulit, ita tamen ut etiam de his praefectis, quos ipse fecerat, triennium nullus impleret... ». Perennis est ici distingué des préfets nommés par Commode, d'autant plus qu'il est resté plus de trois ans en fonction.

145 Dion Cassius, LXXII, 9, 1-2 (Exc. Val. 317).

146 Dion Cassius, LXXII, 10, 1 (Xiphilin, 273). Voir aussi 10, 2 ; 11, 1 ; 12, 1 ; 13, 1.

147 Eusèbe, Hist., V, 21, Perennis est alors présenté comme un juge.

148 M. Absil, op. cit., pp. 184-185 donne une bibliographie qui concerne pour une grande part cet épisode, on peut y ajouter M. Sordi, « Un senatore cristiano dell'eta di Commodo », Epigraphica, 17, 1955, p. 104-112 et F. Grosso, La lotta politica al tempo di Commodo, Turin, 1964, p. 669 et U. Espinosa, « Cómodo y los cristianos : lectura política de las fuentes », Gerion, 13, 1995, p. 127140 et surtout p. 130 .

149 La version en grec des Actes fait de Perennis un proconsul d'Asie, cf. M. Sordi, op. cit., p. 107-108.

150 Hérodien, I, 8, 1. Les chapitres I, 8-9 concernent le gouvernement et la chute de Perennis.

151 Cf. W. Seston et M. Euzennat, « Un dossier ... », 1971 cit. supra, p. 486 (=W. Seston, Scripta Varia, C.E.F.R. 43, Rome, 1980, p. 103). Voir supra.

152 Cf. G. Alföldy, «Der Friedensschluss des Kaisers Commodus mit den Germanen », (=Historia, 20, 1971, p. 84-109) in R. Klein éd., Marc Aurel, Darmstadt, 1979, p. 423 n. 107 désormais dans G. Alföldy, Die Krise des Römischen Reiches, Stuttgart, 1989, p. 59 n. 107. 
valeur de son travail lorsqu'il fut aux affaires, Commode lui laissant de très grandes responsabilités, sur la préservation des intérêts de l'empire durant cette période et finalement sur sa vie tempérée et son caractère incorruptible ${ }^{153}$. C'est aussi une image positive de Pérennis qui émerge d'une remarque de Galien, conservée dans la traduction arabe de son $D e$ moribus, à propos de la fidélité de ses esclaves au moment de la conspiration ${ }^{154}$. Il n'est pas dans notre propos de revenir ici sur les conditions complexes de sa chute et de sa mort et sur le rôle des armées provinciales dans cette affaire. Ses fils, qui étaient entrés au sénat et occupaient alors des gouvernements de province en Illyrie, selon Hérodien, furent eux aussi mis à mort ${ }^{155}$.

\section{AUX CÔTÉS DU PRINCE}

Il est possible d'identifier le préfet du prétoire sur un certain nombre de monnaies $^{156}$ : il se tient derrière l'empereur et tient la main sur son glaive. Ainsi sur les monnaies de 164 célébrant l'avénement de Sohaemus d'Arménie, l'empereur est représenté assis. Trois autres personnes l'accompagnent, debout, sur son tribunal : le préfet est à droite, le glaive bien visible ${ }^{157}$. Il ne peut s'agir que de Furius Victorinus. C'est encore derrière Lucius Verus que l'on peut voir le préfet du prétoire, deux ans plus tard et sur un médaillon, l'empereur est debout sur son tribunal et harangue ses troupes ${ }^{158}$. Est-ce aussi le préfet qui apparaît en 172 sur une monnaie portant la légende " Providentia Aug. »159 ? L'empereur est sur son tribunal, à nouveau dans une scène d'adlocutio, il tient pointée vers le haut une lance, la hasta qu'on lui voit aussi tenir sur certaines scènes de la colonne aurélienne ${ }^{160}$. Derrière lui se tient un personnage de taille comparable, le relief est moins lisible que les autres mais la position de son bras peut correspondre au geste qui amène la main sur le glaive. Incontestablement la monnaie célèbre les victoires militaires récentes qui furent sanctionnées par

153 Dion Cassius, LXXII, 9, 2 et 10, 1.

154 R. Walzer, « New Light on Galen’s Moral Philosophy (from a Recently Discovered Arabic Source) », Classical Quarterly, vol. 43, n¹-2, 1949, p. 82-96 et G. Levi Della Vida, "Two Fragments of Galen in Arabic Translation ", Journal of the American Oriental Society, 70, 3, 1950, p. 182-187.

155 Hérodien, I, 9, 1. Un de ses fils avait gouverné la Lusitanie : CIL II, 258 (ILS, 3939).

156 Nous empruntons très largement ces réflexions à la communication que Claude Brenot présenta le 11 juin 2005 à l'occasion de la table ronde du programme Empire en mutation de l'UMR 8585 au centre Gustave Glotz.

157 W. Szaivert, Die Münzprägung der Kaiser Marcus Aurelius, Lucius Verus und Commodus (161/192), MIR 18, Vienne, 1989, pl. 18 n 28, voir p. 105.

158 W. Szaivert, op.cit., pl. 18, n 24b, voir p. 179.

159 W. Szaivert, op. cit., pl. 18, $n^{\circ} 24$, voir p. 121.

160 Par exemple sur la scène 100 ; Voir J.-M. David, « Les contiones militaires des colonnes trajane et aurélienne : les nécessités de l'adhésion » dans J. Scheid et V. Huet dir., Autour de la colonne Aurélienne, Turnhout, 2000, p. 223 et fig. 120. Sur la signification de cette lance: A. Alföldy, "Hasta - Summa Imperii. The Spear as Embodiment of Sovereignty in Rome ", American Journal of Archaeology, 63-1, 1959, p. 1-27 et pl. 1-10 ; C. Panella, « Emblèmes impériaux découverts sur le Palatin ", dans Col., Rome et les Barbares. La naissance d'un nouveau monde, Venise, 2008, p. 86-91 et 612-614. 
la sixième salutation impériale puis le titre de Germanicus. Ce titre ayant été aussi conféré à Commode, c'est ce dernier qu'il faudrait reconnaître derrière l'empereur selon Jean-Pierre Martin ${ }^{161}$. Il semble difficile d'accepter cette identification : le personnage ne correspond à l'évidence pas à un garçon de 11 ans, et s’il s’agissait de présenter Commode à l'armée, il n'aurait pas été représenté autant en retrait, presque totalement caché par Marc Aurèle. On peut penser qu'il s'agit bien d'un préfet, et sans doute de Bassaeus Rufus.

Dans la première moitié du vingtième siècle, il a été proposé, en particulier par A.L. Frothingham ${ }^{162}$, de reconnaître Bassaeus Rufus sur les reliefs de Marc Aurèle du palais des Conservateurs et de l'arc de Constantin. Ces propositions ont cependant été abandonnées et c'est Claudius Pompeianus que l'on reconnaît désormais dans le personnage qui est associé couramment à Marc Aurèle sur ces reliefs ${ }^{163}$. Les autres portraits figurant sur ces reliefs, en particulier celui de la Liberalitas n'ont pas reçu d'identification faisant l'unanimité164, aucun élément ne les désigne plus particulièrement comme préfet du prétoire. La situation est semblable sur la colonne aurélienne où l'on ne peut identifier un préfet. Ce contraste entre l'imagerie monétaire et les monuments n'est pas surprenant, les deux types d'images n'ont pas exactement la même fonction. Les reliefs commémorent et portent un message qui s'inscrit dans la durée, ils insistent donc plus sur la figure impériale et les enjeux dynastiques que sur l'entourage effectif du prince à un moment donné, en revanche les monnaies qui manifestent l'actualité de la gloire et de l'action du prince sont moins contraintes. Elles témoignent donc fortement de la place du préfet aux côtés de l'empereur.

C'est très significativement que ces trois monnaies concernent aussi des thèmes militaires et guerriers : Furius Victorinus accompagna les empereurs lors des expéditions militaires, puis Macrinius Vindex et Taruttienus Paternus reçurent des commandements et menèrent les troupes au combat, avec plus ou moins de bonheur. La forte implication des préfets du prétoire dans les guerres du règne est aussi signalée par l'Histoire Auguste qui précise que Marc confia le commandement de l'armée à des légats et aux préfets du prétoire: ducentibus etiam exercitum legatis et praefectis praetorio ${ }^{165}$. Peut-on préciser les modalités de cet engagement ?

\section{Place dans l'armée en campagne}

La place du préfet du prétoire, et des troupes ordinairement placées sous son autorité, aux côtés de l'empereur en campagne peut être observée à travers l'œuvre du pseudo-Hygin. Dans son œuvre, dont la datation est discutée,

161 J.-P. Martin, Providentia deorum, recherches sur certains aspects religieux du pouvoir romain, Rome, 1982, p. 333.

162 A.L. Frothingham, " Who built the Arch of Constantine ? III. The Attic. », American Journal of Archaeology, 19, 1, 1915, p. 1-12.

163 I.S. Ryberg, Panel reliefs of Marcus Aurelius, New York, 1967, p. 10-11 et p. 78-79.

164 Voir I.S. Ryberg, op. cit., p. 75 et du même auteur le compte-rendu de J. Brabauw éd., Hommages à Marcel Renard vol. I-III dans AJA, 75, 2, 1971, p. 235-236.

165 S.H.A., Vita Marci, XXII, 2. 
mais que l'on attribue assez souvent au règne de Marc Aurèle ${ }^{166}$, l'auteur délimite au sein de son camp modèle les emplacements réservés aux prétoriens et au préfet du prétoire. Il s'agit alors d'organiser le campement d'une armée en campagne dont on peut se demander dans quelle mesure elle s'approche ou non d'une armée réelle ou «d'un échantillonnage de toutes les unités existant à un moment donné dans l'armée romaine considérée dans son ensemble ${ }^{167}$. Quoi qu'il en soit, l'auteur place les prétoriens dans la partie centrale du camp, qui se nomme précisément le prétoire. Groupés par cohortes, ils disposent d'un emplacement plus vaste que les autres soldats, leurs tentes étant plus grandes ${ }^{168}$. Les cavaliers prétoriens sont groupés un peu à part dans le côté droit du prétoire, non loin des equites singulares augusti ${ }^{169}$. Enfin, à proximité, "immédiatement adjacent à la voie principale” un emplacement est réservé au préfet du prétoire, jouxtant ceux des comites augusti ${ }^{170}$. Lorsqu'il élabore le plan d'un camp type, le pseudoHygin donne un ordre d'idée quant au nombre des cohortes de prétoriens, nombre variable au demeurant : il en retient quatre, ce qui fait deux mille hommes environ ${ }^{171}$, quatre cents cavaliers prétoriens étant compris dans ce nombre. Il reste à leur ajouter quatre cent cinquante equites singulares, ce qui porte le total à près de deux mille cinq cents hommes . Ce qui fait une troupe finalement modeste, environ $5 \%$ des plus de quarante mille hommes que compte ce camp type. Si l'on s'en tient à l'ordre d'idée proposé par le Pseudo-Hygin on ne peut faire des troupes du prétoire le coeur de l'armée de campagne, et leur nombre restreint comme leur emplacement convient d'abord à leur rôle essentiel, la protection de l'empereur et de son entourage. En outre, on ne saurait négliger les missions administratives qui pouvaient être remplies par les soldats de l'officium du préfet ${ }^{172}$. Le voisinage des prétoriens avec les primipilaires et les évocats ${ }^{173}$ peut aussi laisser penser à un rôle d'encadrement. Ils sont enfin parfois considérés comme des troupes de choc, un corps d'élite destiné à guider et appuyer le gros des troupes ${ }^{174}$. Il faut avec J.B. Campbell relativiser cette

166 Cf. Pseudo-Hygin, Des fortifications du camp, éd. et trad. M. Lenoir, C.U.F., Paris, 1979, plus particulièrement p. 111-133 à propos de la date du texte, l'époque de Trajan selon M. Lenoir. S’il a effectivement raison, il nous semble cependant encore possible de considérer ce document comme illustrant la participation quantitative qu'on pouvait attendre du prétoire au deuxième siècle lors de grandes campagnes.

167 Pseudo-Hygin, op. cit., p. 112. Des deux hypothèses M. Lenoir opte résolument pour la seconde. De notre point de vue la première semble pourtant digne d'être considérée.

168 Pseudo-Hygin, op. cit., 6.

169 Pseudo-Hygin, op. cit., 7.

170 Pseudo-Hygin, op. cit., 10.

171 Cf. Pseudo-Hygin, op. cit., éd. et trad. M. Lenoir, C.U.F., Paris, 1979, § 30 et p. 56, nº 47. La taille des cohortes au deuxième siècle a été discutée, nous considérons qu'elles sont quingénaires, voir Y. Le Bohec, L'armée romaine, Paris, 1990, p. 21.

172 Cf. J. Nélis-Clément, op. cit., p. 94-106 et surtout p. 101-103.

173 Pseudo-Hygin, op. cit., 6.

174 Cf. B. Rankov, The Praetorian Guard, Oxford, 1994, p. 14 qui parle de « genuine fighting elite ». Destiné avant tout au grand public, l'ouvrage offre une synthèse commode quant à l'équipement des prétoriens et à leurs représentations. A. Passerini dans son ouvrage contemporain de celui de M. Durry, Le coorti pretorie, Rome, 1939, voyait en eux l'élite des combattants ; voir Y. Le Bohec, L'armée romaine, Paris, 1990, p. 20-21. 
conception $^{175}$ : le nombre limité de prétoriens et leur position autour de l'empereur n'incitent pas à supposer un impact stratégique ou tactique particulier.

On a pu chercher dans les monuments figurés du règne un autre témoignage. On a alors l'impression d'un contraste assez net avec l'importance numérique limitée des prétoriens dans les armées de campagne que l'on peut mettre en évidence si l'on considère le texte du Pseudo-Hygin. Les prétoriens ont en effet souvent été reconnus dans les reliefs historiques. L'interprétation de ces reliefs est cependant délicate. Un passage de Dion Cassius $^{176}$ nous apprend que les prétoriens portaient, à l'époque de Macrin, la cuirasse à écailles, la squamata. Appliquant cette information aux reliefs de la colonne aurélienne, E. Petersen et A. von Domaszewski voulurent reconnaître des prétoriens dans les porteurs de cette cuirasse, fantassins ou cavaliers (equites praetoriani). Critère distinctif auquel ils ajoutèrent le port du gladius à droite, pendu au baudrier, et le port des femoralia ${ }^{177}$. Les enseignes, en particulier l'une se terminant par un aigle et l'autre par une main ouverte ${ }^{178}$, pourraient aussi indiquer des prétoriens. L'association de porteurs de squamata à ces enseignes, mais aussi leur situation dans l'ordre de marche de l'armée et leur présence fréquente à proximité de l'empereur a pu sembler confirmer la thèse de Petersen et Domaszewski ${ }^{179}$. Il nous semble cependant difficile de l'accepter systématiquement. D'une part car on ne peut pas considérer les reliefs historiques romains comme des témoignages exacts. Dans les représentations qu'ils nous offrent la part des conventions est immense ${ }^{180}$ et la disposition des troupes et des costumes ${ }^{181}$ répond à des considérations esthétiques et idéologiques ${ }^{182}$ bien plus qu'à un désir de fidélité, si bien que l'on a pu parler de la fantaisie de cet art romain $^{183}$. L'alternance de costumes et d'équipements différents a pu être prisée des sculpteurs, heureux de trouver dans ces différences des moyens d'éviter la monotonie pour l'œil. Plusieurs scènes de la colonne associent les trois types de cuirasse, squamata, segmentata et cotte de mailles ${ }^{184}$.

175 J.B. Campbell, The emperor and the Roman army 31 BC - AD 235, Oxford, 1984, p. 114 : « there is no sign that the Praetorians were developed to be an elite strategic striking force ».

176 Dion Cassius LXXVIII (LXXIX), 37, 4 (E. Cary, p. 424-425).

177 Cf. M. Durry, Les cohortes, cit. supra, p. 227-228 ; voir aussi B. Rankov, op. cit., p. 23 et 59.

178 Cf. M. Durry, op. cit., p. 200-205, en particulier, p. 201 n. 7.

179 M. Durry, op. cit., p. 228 et p. 233.

180 Ainsi à propos des casques des prétoriens voir les remarques de B. Rankov, op. cit., p. 19-21 et plus généralement $\mathrm{M}$. Galinier, "La représentation iconographique du légionnaire romain », Légions de Rome, Lyon, 2000, p. 422-425.

181 La notion d'uniforme était étrangère à l'armée romaine, ce qui, en soi complique l'identification de tel ou tel corps de troupes, même si à défaut d'uniforme une certaine uniformité devait exister dans l'équipement, voir M. Feugère, Les armes des Romains, Paris, 2002, p. 226-227.

182 Voir les réflexions de M. Galinier, op. cit., pp. 422-425. Voir aussi J. Ch. Balty, « L’armée de la Colonne Aurélienne : images de la cohésion d'un corps », in J. Scheid et V. Huet dir., Autour de la colonne Aurélienne: Geste et image sur la colonne de Marc Aurèle à Rome, (désormais abrégé Colonne) Turnhout, 2000, p. 197-203, même si le diagnostic porté p. 202 sur l'armée romaine à l'époque de Marc Aurèle nous semble un peu trop sombre.

183 M. Durry, op. cit., p. 232-236. Voir aussi M. Galinier, op. cit., p. 417.

184 Ainsi, scènes 8 et 9 (Colonne, fig. 22 et 23) ou scène 31 (Colonne, fig. 50). Cf. M. Galinier, op. cit., p. 422. 
D'autre part car nous savons que des légionnaires pouvaient porter l'équipement décrit comme caractéristique des prétoriens par Petersen et Domaszewski. La squamata est bien attestée pour des fantassins légionnaires, en particulier pour des centurions, même si son usage a sans doute été assez restreint en raison de son utilisation finalement peu commode ${ }^{185}$. Il semble donc en fait plus que difficile d'espérer distinguer les prétoriens des légionnaires sur la base de leur équipement ${ }^{186}$, en l'absence de signes distinctifs comme des enseignes ou le scorpion. Malheureusement ce dernier semble être totalement absent des reliefs nous concernant, que ce soit la colonne ou ceux de l'arc de Constantin et du palais des Conservateurs ${ }^{187}$. Les enseignes au moins semblent nous assurer que la garde était présente lors des opérations militaires représentées sur les reliefs, mais l'était-elle entièrement comme le pensait A. von Domaszewski ${ }^{188}$, ou seulement partiellement comme semble en témoigner le pseudo-Hygin? Si la présence des enseignes nous indique des prétoriens, le contexte de la scène peut nous aider à les reconnaître comme lorsque l'armée en marche est représentée ${ }^{189}$, ou à proximité de l'empereur. La colonne, au coeur de Rome, pouvait alors rappeler la place que les prétoriens occupaient auprès de l'empereur et les liens qui les unissaient à sa personne, en temps de paix à Rome comme en temps de guerre au loin sur les frontières. Elle était aussi un message adressé par le prince aux troupes de sa capitale, mais ne saurait cependant réellement nous renseigner sur les modalités d'engagement des troupes prétoriennes lors des combats contre les barbares.

\section{Commandement au combat}

Les préfets commandèrent des troupes lors d'opérations importantes. Des prétoriens se trouvaient sans doute dans ces troupes, mais ils ne pouvaient en constituer l'essentiel du point de vue quantitatif. Nous avons vu aussi que nous avons peu d'indices pour leur attribuer un rôle tactique particulier, ou pour éclairer ce que l'on appellerait aujourd'hui leur doctrine d'emploi. Il

\footnotetext{
185 Cf. M. Feugère, Les armes des Romains, Paris, 2002, p. 123-127.

186 B. Rankov, op. cit., p. 23-24. ; M. Galinier, op. cit., p. 422.

187 Contrairement à ce que pensait A. von Domaszewski, dans son ouvrage sur les enseignes de l'armée romaine, rien ne prouve que les soldats apparaissant sur les panneaux soient nécessairement des prétoriens, cf. I. Scott Ryberg, Panel Reliefs of Marcus Aurelius, New York, 1967, p. 62 n. 4. On reconnaît en général un prétorien dans le soldat revêtu de la squamata figurant sur le relief du Palais des Conservateurs interprété comme représentant la Clementia par I. Scott Ryberg ou la Mansuetudo par B. Andreae, L'art romain ${ }^{2}$, Paris, 1998, n 556 ; cf. I. Scott Ryberg, op. cit., p. 11 n. 6 ; B. Rankov, op. cit., p. 58. Des soldats portant le même genre de cuirasse, et donc souvent considérés comme des prétoriens se retrouvent sur les reliefs de la profectio et de l'adlocutio; cf. I. Scott Ryberg, op. cit., p. 34-35 et 53. Leur présence aux côtés de l'empereur, plus que leur équipement rend cette identification plausible, mais dans la scène de l'adlocutio les signa sont ceux de légions, cf. M. Galinier, op. cit., p. 422. Enfin une enseigne prétorienne semble se trouver dans le relief de la lustratio et dans celui de la soumission des barbares ; cf. I. Scott Ryberg, op. cit., p. 40 et 62.

188 Cf. M. Durry, op. cit., p. 201 n. 3. À propos des enseignes figurant sur la colonne, il faut noter le scepticisme de M. Galinier, "La représentation iconographique du légionnaire romain », Légions de Rome, Lyon, 2000, p. 425.

189 Ainsi, scènes 1,3 et 78 (Colonne, fig. 1, 3, 11-13, 98).
} 
est nécessaire en tout cas de penser qu'à leurs côtés, sous les ordres du préfet, on trouvait les autres catégories de troupes : légionnaires, auxiliaires, alliés et troupes de renforts. La présence des premiers de cette liste se déduit de leur importance numérique dans les troupes rassemblées pour la guerre et de leur présence dans tous les ordres de marche ou de bataille connus pour notre époque. Leur présence en nombre important soulève cependant une question. Le commandement des corps de troupes légionnaires revenait normalement à des sénateurs. Était-il possible de placer ces derniers, dans le cadre d'une armée de campagne, sous les ordres d'un préfet du prétoire, personnage certes éminent, mais qui restait cependant membre de l'ordre équestre ? Comment pouvait alors s'organiser le commandement, selon quelle préséance ? Un légat clarissime de rang prétorien pouvait-il se placer, avec sa légion, sous les ordres du préfet ? Cela ne semble pas impossible. Les ornements consulaires pouvaient conférer au préfet une dignité supérieure à celle du légat et par ailleurs le préfet représentait l'autorité de l'empereur, ses compétences, leur extension et leur définition, dépendant en dernier ressort de la volonté du prince. Il semble possible de se demander si l'emploi de vexillations légionnaires regroupées plutôt que de légions entières n'a pas pu être un moyen de diminuer les susceptibilités des gradés clarissimes $^{190}$, et éventuellement des soldats légionnaires. Les vexillations peuvent en effet être dirigées par des centurions ${ }^{191}$, des primipiles ${ }^{192}$, des préfets de camp ou des officiers appartenant à l'ordre équestre ayant le titre de praepositus. C'est ce titre, associé à celui de procurateur, que porte L. Iulius Vehilius Iulianus, officier équestre aux capacités militaires éprouvées, lors de la seconde expédition germanique, où précisément le commandement de Paternus eut une si grande importance ${ }^{193}$. Nous ignorons malheureusement la composition de ces troupes et nous n'avons donc pas la certitude qu'il ne s'agissait pas d'auxiliaires. Nous savons en revanche avec certitude que les détachements commandés, à peu près au même moment, par T. Plautius Felix Ferruntianus étaient composés de légionnaires, Plautius accomplissant alors ses milices équestres ${ }^{194}$. Il est aussi possible de penser aux légionnaires tirés des légions II et III Italica qui construisirent en 169 une partie des murailles de Salone en étant placés sous la responsabilité

190 Nous ne supposons pas ici une rivalité des deux ordres, mais une émulation, une lutte, au sein des plus grands serviteurs de l'état, autour de leurs prérogatives habituelles, où chacun cherche à défendre et augmenter son rang et sa dignité, en s’appuyant éventuellement sur les spécificités de son ordre.

191 Par exemple CIL III, 14433 (ILS, 9118) ; cf. R. Saxer, Untersuchungen zu den Vexillationen des römischen Kaiserheeres von Augustus bis Diokletian, Cologne, 1967, n 72, p. 41-42.

192 Sous Septime Sévère : CIL X, 6657 ; cf. R. Saxer, op. cit., n 75-76, p. 43-44.

193 CIL VI, 31856 = 41271 ; Cf. CPE, 180 ; PME I, 136 et t. IV p. 1620 ; R. Saxer, op. cit., $\mathrm{n}^{\circ} 67, \mathrm{p} .35$.

194 CIL VIII, 619 = 11780 (ILS 2747). Plusieurs aspects de cette inscription ont suscité une bibliographie importante et ont été discutés. Cf. CPE 198 et Suppl., p. 53 ; PME, P 41 et t. IV, p. 1681. Sur ses vexillations R. Saxer, op. cit., $\mathrm{n}^{\circ} 74$, p. $42-43$ et surtout sur les soldats de la III Augusta qu'il dirigea voir G. Di Vita-Evrard, « Légionnaires africains en Pannonie au Ilème siècle ap. J.-C. », in G. Hajnoczi (ed.), La Pannonia e l'Impero Romano, Atti del convegno internazionale « La Pannonia e l'Impero Romano », Accademia d'Ungheria e l'Istituto Austriaco di Cultura Roma, 13-16 gennaio 1994, Milan, 1995, p. 97-114. 
d'un centurion de la légion II Traiana195. Il était donc en théorie possible de créer une chaîne de commandement permettant l'intégration de vexillations légionnaires sans officier clarissime au sein d'un corps d'armée dirigé par un préfet du prétoire. Il ne faut cependant pas oublier qu'il n'était pas seul alors à diriger de tels regroupements de troupes. Des légats sénatoriaux avaient aussi de grands commandements. Par ailleurs les sénateurs ne cessèrent pas de diriger des légionnaires regroupés en vexillation. Cela est bien attesté tout au long du règne pour des légats de rang prétorien comme Iulius Geminius Marcianus, Vettius Sabinianus Iulius Hospes et Valerius Maximianus ${ }^{196}$. Enfin, quelle taille supposer pour ces corps de troupes dirigés par les préfets ? Les indices sont rares. Quoi qu’il en soit il nous faut surtout constater l'étendue des lacunes de nos connaissances quant aux modalités d'engagement des préfets de Marc Aurèle commandant des troupes aux combats, à savoir au moins Macrinius Vindex et Taruttienus Paternus.

\section{Le conseiller militaire le plus proche du Prince?}

Le rôle des préfets durant les campagnes fut important, mais doit être abordé avec prudence: si nous pouvons affirmer l'importance de ce rôle, ses modalités exactes nous échappent. On ne saurait faire du préfet un « chef d'état-major ». Ce serait projeter sur sa personne et ses fonctions des notions modernes et manquer, pour une analogie facile et séduisante, ce qui fait la spécificité de sa place et de ses fonctions. L'organigramme de l'armée romaine s'éloigne nécessairement de celui des armées modernes quand bien même elle devait faire face aux mêmes défis militaires intemporels (logistique, renseignement, communication... ). Il est certain que l'empereur pouvait confier ponctuellement à son préfet un commandement militaire très important, comme ce fut le cas pour Macrinius et Taruttienus, mais alors il s'agit plus de commander un corps d'armée que d'administrer toutes les troupes. Il est certain aussi qu'un empereur pouvait se révéler velléitaire et très largement déléguer, permettant à son préfet de s'occuper d'affaires militaires se déroulant dans les provinces, comme Commode le fit avec Perennis. Mais nous sommes là dans un cas particulier, extrême ${ }^{197}$ et cela est bien sûr insuffisant pour systématiser une position et des fonctions. Il est donc difficile de dire si les attributions militaires des préfets de Marc Aurèle anticipaient sur ce que l'on peut constater à une époque plus basse. Ainsi nous avons vu comment les passages de l'Histoire Auguste mettant un de nos préfets en relation avec le ravitaillement des troupes peuvent être tenus plutôt pour des anachronismes que pour des témoignages dignes de foi. Il faut nous garder de projeter sur notre époque, ou même sur le début du troisième siècle, des attributions qui résultaient d'un long processus et d'une situation complexe.

195 CIL III, 1980 (ILS, 2287) ; cf. R. Saxer, op. cit., n 71, p. 40-41.

196 Voir CIL VIII, 7050 (ILS, 1102) et I.L. Afr., 281 et (AE 1956, 124).

197 Cf. J.B. Campbell, op. cit., 1984, p. 115. 
Mais ne pas systématiser le rôle militaire du préfet et refuser d'envisager des fonctions militaires formellement définies et continuellement exercées autres que le commandement du prétoire ne doit pas mener à sous-estimer le rôle qu'il pouvait jouer dans la prise de décisions militaires. Le commandement qu'il exerçait sur les prétoriens, l'expérience qu'il pouvait avoir, et pour laquelle on l'avait choisi, mais surtout sa présence presque continuelle aux côtés du prince et la relation de confiance qui déterminait cette présence, le préfet étant garant de la sécurité et de la vie du prince, voilà autant d'éléments qui devaient peser lorsqu'une question d'ordre militaire était abordée et que le préfet donnait son avis. Il est à cet égard évident que si Marc Aurèle fit le choix de préfets ayant des profils de carrières militaires c'est qu'il attendait d'eux des conseils dans ce domaine et une prise de responsabilité importante. Mais on ne saurait oublier non plus que, même alors, le préfet agit parmi d'autres conseillers du prince : les comites, les legati, c'est-à-dire des gens comme Claudius Pompeianus ou, à un niveau moindre, Pertinax. Ils étaient eux aussi des experts militaires qui devaient être écoutés et pour certains aussi des intimes du prince. Nous voudrions à cet égard constater que les responsabilités militaires de Taruttienus à la fin du règne ne sont pas séparables de ses liens avec de puissants membres de l'ordre sénatorial, proche du prince et eux aussi placés à la tête d'armées importantes: Pompeianus bien sûr, mais aussi Salvius Iulianus ${ }^{198}$. Si Taruttienus est écouté, s’il reçoit des responsabilités militaires décisives, c'est qu'il n’est pas seul mais intégré à un réseau partageant sans doute des intérêts communs et défendant vraisemblablement les mêmes avis stratégiques. Même s’il n'est pas possible de cerner, pour chacun des préfets de Marc Aurèle, ce réseau de relations que l'on devine autour de Taruttienus Paternus et cette conjonction de facteurs qui fit que son avis était entendu, il nous semble nécessaire de penser que la situation était similaire dans d'autres cas. Furius Victorinus pesa sans doute lourdement dans les décisions militaires du début du règne. D’une part son collègue ne pouvait en effet guère prétendre à une expérience militaire importante, d'autre part les deux empereurs étaient eux-mêmes peu expérimentés. On comprend qu'il ait accompagné Vérus en Orient et surtout qu'il ait été décoré à son retour. Si un commandement fut confié lors d'une bataille à Vindex c'est sans doute qu'il avait lui aussi une position semblable. Enfin, Bassaeus Rufus, tant par la durée de sa présence en poste que par la connaissance intime de l'armée qu'il avait, fut nécessairement un conseiller écouté de Marc Aurèle en même temps qu'un responsable des plus importants. Comme ses collègues, il se trouvait au sein d'un groupe de commandement d'où les décisions pouvaient émerger collégialement avant d'être avalisées par l'empereur sans que ce groupe soit pour autant explicitement structuré en fonction de missions bien définies, comme le sont nos modernes états-majors et sans que ce groupe soit toujours clairement et explicitement séparé du reste de la cour impériale.

198 Dion Cassius LXXII (LXXIII), 5, 1-2. 
Rôles des préfets du prétoire de Marc Aurèle

L'examen des commandements que reçurent les préfets de Marc Aurèle lors de ses campagnes amène donc à considérer le rôle et les fonctions de nos personnages. Cela a fait l'objet de plusieurs études qui ont cherché à préciser leurs actions dans les domaines militaires, judiciaires et politiques ${ }^{199}$. Pour ces trois aspects le règne de Marc Aurèle est relativement bien documenté, plusieurs sources nous renseignant sur l'activité des préfets et correspondant en général à ce que l'on a pu déduire à partir des autres règnes. Cependant, il est clair que le caractère général du règne de Marc Aurèle, la présence, la longueur et la difficulté des guerres a fortement influencé et modifié le rôle des préfets, en confiant une part accrue à leur responsabilité militaire. Ce caractère particulier se retrouvant au demeurant dans les carrières des préfets, dont le déroulement et la nature peuvent nous aider, en partie, à mieux cerner le choix du prince de s'associer tel ou tel personnage en tant que préfet. En effet l'examen des carrières montre que les impératifs militaires ont assez nettement dirigé le choix des différents personnages, parmi un ensemble de candidats assez restreint cependant, le sommet de l'ordre équestre étant finalement un petit monde. Seul Cornelius Repentinus fait exception, mais il avait été choisi avant le déclenchement des conflits. Inversement Bassaeus Rufus, personnage sorti du rang, symbolise le mieux cette recherche d'hommes compétents dans le domaine des armes. Néanmoins les différentes carrières ne sont pas semblables, et certaines sont très mal connues. Même dans ce dernier cas nous savons que Flavius Constans et Macrinius Vindex avaient gouverné au moins une province frontière ayant une garnison d'auxiliaires. Cela avait été aussi le cas pour Bassaeus Rufus. En revanche c'est à la tête des flottes que Furius Victorinus avait prouvé ses capacités martiales avant d'intégrer les offices palatins. Nous ne pouvons que regretter d'ignorer les débuts de la carrière de Taruttienus.

Cependant les impératifs militaires n’ont jamais totalement exclu les autres critères de choix et notamment celui des compétences administratives et juridiques. C'est ce qui ressort clairement des activités de Taruttienus Paternus et d'un passage de l'Histoire Auguste mentionnant leur présence aux côtés de l'empereur et l'importance de leur autorité lorsque ce dernier disait le droit ${ }^{200}$. Il est vrai aussi que les guerres n'empêchèrent pas les préfets de Marc Aurèle d'assumer un certain nombre de missions

199 Dans le cadre de l'étude des cohortes du prétoire et de leur préfet les deux ouvrages importants, et quasi contemporains, de M. Durry, Les cohortes prétoriennes, Paris, 1938 et de A. Passerini, Le coorti pretorie, Rome, 1939 restent des références. L'importante étude de L. L. Howe, The Praetorian Prefecture from Commodus to Diocletian, Chicago, 1942, sur laquelle on lira toujours avec profit le compte rendu de O.W.Reinmuth dans The American Journal of Philology, 65, 2, 1944, p. 196-200, ne concerne cependant notre période qu'à la marge . Plus récemment, des synthèses brèves et complémentaires ont été proposées par F. Millar, The Emperor in the Roman World ${ }^{2}$, Londres, 1992 (première édition 1977), p. 122-131 (privilégiant l'aspect judiciaire) et par J.B. Campbell, The emperor and the Roman army 31 BC - AD 235, Oxford, 1984, p. 114-120 (privilégiant l'aspect militaire). Elles sont ignorées de M. Absil, Les préfets du prétoire d'Auguste à Commode, Paris, 1997, qui est parfois imprécis et décevant.

200 S.H.A., Vita Marci, X, 10. 
administratives ou judiciaires. Ces missions pouvaient résulter d'une délégation de pouvoir spécifique et ponctuelle par l'empereur ${ }^{201}$.

La fameuse inscription de Saepinum ${ }^{202}$ constitue un témoignage essentiel sur la place des préfets du prétoire dans l'administration, elle soulève cependant nombre de questions. C'est sur la demande des affranchis impériaux Cosmus ${ }^{203}$, secrétaire a rationibus, et Septimianus, un de ses subordonnés, que les préfets interviennent pour protéger les «fermiers des troupeaux de moutons » dont la transhumance était perturbée par les stationarii et les magistrats de Saepinum et de Bovinum. Pour faire cesser les dommages causés au fisc (magna fisci iniuria) qui en résultaient, les préfets admonestèrent les autorités en question et les menaçèrent d'une enquête plus approfondie. Leur lettre, celle de Cosmus et celle de Septimianus furent gravées à Saepinum afin de monumentaliser cette intervention. La protection des drailles des Abruzzes incombait donc, in fine, à l'autorité des préfets du prétoire, et requérait leur attention même en temps de préparatifs de guerre. La nature exacte du préjudice subi par le fisc est l'un des points les plus débattus. S'opposant à la lecture la plus courante, Mireille Corbier a proposé de voir dans ce préjudice non pas la perte de moutons appartenant aux troupeaux de l'empereur, mais le manque à gagner, occasionné par les troubles, sur les taxes de transhumance ${ }^{204}$. Elle n'a pas cependant convaincu tous les tenants de la thèse traditionnelle 205 . Quoi qu'il en soit la lecture du dossier montre les échelons inférieurs de l'administration incapable de régler un problème local, l'intervention des préfets du prétoire semble alors assez ordinaire. C'est Septimianus, affranchi impérial adjoint de l'a rationibus qui a suggéré à son coaffranchi et supérieur, Cosmus secrétaire a rationibus, d’avertir les préfets du prétoire puisque les magistrats locaux et le stationarii ne tiennent pas compte de ses injonctions : la procédure semble, pour lui, aller de soi. La mention de stationarii est importante. Ils pourraient être des soldats chargés d'une mission de police à l'image des autres stationarii chargés de telles missions en Italie au troisième siècle ou des milites stationarii mentionnés dans une lettre de Marc Aurèle et Commode206: il pourrait alors s'agir de prétoriens ${ }^{207}$. L’intervention des préfets du prétoire serait alors facile à

201 Cf. F. Millar, op. cit., p. 123-124 ; M. Absil, op. cit., p. 76

202 CIL IX, 2438 = $4916 ;($ AE 1983, n 331).

203 Cf. CIL VI, 455 ; ( $A E$ 1995, n 59=91) datée de la fin septembre 168.

204 M. Corbier, « Fiscus and Patrimonium. The Saepinum Inscription and Transhumance in the Abruzzi », JRS, 73, 1983, p. 126-131 ; Ead. « La transhumance entre Samnium et Apulie : continuité entre l'époque républicaine et l'époque impériale ", La romanisation du Samnium aux Ilème et Ier siècles av. J.-C., Naples, 1991, p. 172-176; Ead., Donner à voir, donner à lire. Mémoire et communication dans la Rome ancienne, Paris, 2006, p. 217-232.

205 Voir ainsi P.-A. Brunt, Roman Imperial Themes, Rome, 1990, p. 427-428 ; E. Lo Cascio, « I « greges ouiarici » dell'iscrizione di Sepino (CIL IX, 2438) e la transumanza in età imperiale » dans Scritti offerti a Ettore Paratore ottuagenario, Abruzzo. Rivista dell'Istituto di studi abruzzesi, XVIIIXXVIII, 1985-1990, repris dans E. Lo Cascio, Il princeps e il suo impero. Studi di storia amministrativa e finanziaria romana, Bari, 2000, p. 151-161 ; H. Galsterer, «Local and provincial institutions and government », The Cambridge Ancient History2, XI, Cambridge, 2000, p. 346 n. 9.

206 Digeste XI, 4, 1, 2.

207 Voir M. Corbier, « La transhumance » 1991, cit. supra, p. 169-170 ; F. Millar, « Italy and the Roman Empire : Augustus to Constantine », Phoenix, 40, 3, p. 295-318 et surtout 312-313. 
comprendre: eux seuls pouvaient réprimander leurs subordonnés qui s'étaient entendus avec les magistrats locaux au détriment des bergers transhumants. Cela signifierait aussi que l'autorité des préfets s'exerçait en Italie et qu'ils étaient déjà " les détenteurs du pouvoir coercitif en Italie » 208 au-delà des cent miles qui bornaient l'autorité du préfet de la Ville comme cela est mieux attesté au troisième siècle ${ }^{209}$. Si cette hypothèse nous semble possible, l'identité des stationarii de Saepinum n'est cependant pas univoque et ils n’ont pas été en général interprétés comme des prétoriens mais comme des personnes dépendants des autorités municipales ${ }^{210}$. Dans ce dernier cas l'inscription ne fournit pas clairement les raisons pour lesquelles on fait appel au préfet ${ }^{211}$.

Le dossier de Saepinum n'offre donc pas de réponse claire aux questions que l'on peut se poser sur le rôle administratif et judiciaire des préfets du prétoire en Italie et ne permet pas de dire si l'on avait sous Marc Aurèle une situation aussi institutionnalisée qu'elle semble l'être au troisième siècle. Toutefois il nous permet de voir les préfets en action et de comprendre quels éléments firent que peu à peu ils se retrouvèrent chargé du maintien de l'ordre en Italie: ils dirigeaient les troupes les plus importantes de la péninsule - qui en était presque dépourvue par ailleurs -, et pouvaient recevoir de nombreuses fonctions par délégation du prince. L'exercice d'une juridiction découle donc en partie des compétences militaires des préfets qui commandent alors les troupes présentes en Italie.

On peut penser, avec F. Millar, que c'est aussi le rôle de protection de l'empereur et de commandant des cohortes, qui fut à l'origine, par extension, des autres fonctions judiciaires ${ }^{212}$ et en particulier de la présence active du préfet aux côtés du prince lors de procès importants. Bassaeus Rufus fournit là encore un bon exemple, puisque nous avons vu qu'il est présent aux côtés de Marc Aurèle lors du procès d'Herode Atticus à Sirmium, et qu'il ne se prive pas d'intervenir ${ }^{213}$. Il faut noter au passage ce paradoxe documentaire qui fait que nous connaissons mieux pour Bassaeus Rufus ses activités judiciaires et administratives que militaires. Si l'on en croit l'Histoire Auguste cependant, Marc Aurèle avait exclu ses préfets des procès où un sénateur était jugé pour crime capital : seuls des sénateurs pouvaient prendre part à ces procès ${ }^{214}$. Cela correspond à ce que nous savons du déroulement de l'épuration consécutive à la révolte d’Avidius

\footnotetext{
208 M. Corbier, « La transhumance », 1991, cit. supra, p. 171.

209 F. Millar, op. cit.

210 E. Lo Cascio, op. cit., p. 151 n. 3.

211 H. Galsterer, op. cit. En dernier lieu sur la question des stationarii voir M. Corbier, Donner à voir, donner à lire. Mémoire et communication dans la Rome ancienne, Paris, 2006, p. 228 qui conserve, à juste titre à notre avis, son hypothèse.

212 Mais on ne peut à notre avis tirer argument de cela pour restreindre l'essentiel de l'activité judiciaire du préfet aux cas « où la sûreté de l’État était en cause » (M. Absil, op. cit., p. 81), sauf à avoir de ces termes une définition suffisamment large pour y inscrire les problèmes de transhumance. L'essentiel des responsabilités judiciaires n'est pas nécessairement composé de celles qui ont le plus de retentissement.

213 Philostrate, Vie des sophistes, II, 1, 11 (561) (éd. Wright, p. 171). Voir la bibliographie indiquée supra à propos de Bassaeus Rufus.

214 S.H.A., Vita Marci, X, 6.
} 
Cassius. Marc se montra clément et prudent, tout en s'appuyant sur le sénat pour mener les procès 215 . Enfin, plus ordinairement que ces grands procès, les préfets étaient présents au consilium principis avec les autres grands dignitaires de l'ordre équestre, comme en témoigne la table de Banasa ${ }^{216}$.

Importants conseillers et adjoints militaires de l'empereur, les préfets, hommes de confiance de l'empereur, reçurent aussi de Marc Aurèle une autorité judiciaire et administrative qui s'exerçait de manière assez diversifiée et régulière. La guerre orienta le choix des personnes retenues pour ce poste, marqua profondément leur activité, mais n’oblitéra jamais totalement une activité plus quotidienne et moins martiale. Bénéficiant de la confiance de l'empereur et d'une position influente dans la marche quotidienne des affaires de l'empire, les préfets étaient donc des personnalités politiques de premier plan.

\section{LES PREFETS AU SEIN DU PERSONNEL POLITIQUE DIRIGEANT L'EMPIRE}

Nous l'avons dit, le choix du prince ne pouvait s'effectuer que dans un groupe d'individus finalement assez restreint: le préfet devait être choisi parmi les chevaliers ayant accompli une carrière la plus complète possible. Le poste constituait le couronnement d'une carrière, la dignité suprême de l'ordre équestre. L'empereur ne pouvait donc choisir le préfet, sauf à courir le risque de susciter des jalousies importantes, que parmi les membres les plus honorables de l'ordre, en respectant dans la mesure du possible la hiérarchie des dignités induites par l'existence de fonctions hiérarchisées et la plus ou moins grande ancienneté dans ces fonctions. Le rappel précipité de Bassaeus Rufus d'Égypte vers Rome montre pourtant que le choix impérial primait et que l'on n’hésitait pas à brusquer le déroulement logique des fonctions si le besoin s'en faisait ressentir. Ce rappel peut aussi montrer qu'il existait peut-être l'équivalent d'une présélection, implicite et informelle. On peut en tout cas penser que lorsqu'il choisissait les titulaires des plus hautes fonctions équestres - chancellerie et grandes préfectures l'empereur savait qu'il orientait la carrière du personnage vers une éventuelle préfecture du prétoire si le poste devenait vacant.

Lorsque les guerres ou la peste ne suppriment pas le titulaire de la fonction, on constate une assez grande stabilité en poste, au moins neuf ans pour Bassaeus Rufus, peut-être autant pour Tarrutienus, un peu moins pour Furius Victorinus. Cela suppose un lien de confiance fort entre Marc Aurèle et ses préfets, et la capacité de bien gérer le devenir des autres chevaliers haut placés. Leur carrière se trouve alors bloquée. L'adlection permet de récompenser ceux pour qui la voie était désormais bouchée mais que l'on désirait honorer, où dont l'on désirait exploiter les compétences. On peut penser à Volusius Maecianus, Varius Clemens, Aius Sanctus ${ }^{217}$ ou bien encore au quasi-anonyme -ilius ${ }^{218}$. Pourtant le tableau d'avancement logique

\footnotetext{
215 S.H.A., Vita Marci, XXV, 5-8.

216 I.A.M., 94. Étrangement M. Absil, op. cit., p. 77-80 discute de la place des préfets dans le consilium sans même mentionner ce document.

217 (AE 1961, 280).

218 CIL VI, 1564 (ILS 1452) ; CIL VI (8,3), 41130 (Rome). Cf. CPE nº 178.
} 
est loin d'être toujours respecté, ce qui est normal pour un poste à si hautes responsabilités: l'ancienneté ne suffit pas à garantir l'accession à la préfecture. La soudaine ascension de Cornelius Repentinus suscita, nous l'avons vu, bien des rumeurs. Un peu plus tard, il est plus que probable que Taruttienus est passé devant Calvisius Statianus, immobilisé dans un long gouvernement égyptien. Il est même possible de penser que Paternus ne passa pas par l'exercice d'une grande préfecture romaine. Il est clair en tout cas que pour Marc Aurèle le gouvernement de l'Égypte n'était pas un passage obligé pour ses préfets, seuls Furius Victorinus et Bassaeus Rufus gouvernèrent la province.

Enfin l'appartenance à l'élite de l'ordre équestre pour les candidats au poste, ce choix personnel par l'empereur puis la durée en fonction favorisaient incontestablement la constitution d'un important et vaste réseau de clientèles et de recommandations, ainsi que l'élévation du rang d'une bonne partie de la famille et des dépendants. Cornelius Repentinus, Bassaeus Rufus, Macrinius Vindex, Taruttienus Paternus et Tigidius Perennis eurent tous des enfants qui entrèrent dans l'ordre sénatorial soit directement pour les garçons, soit en épousant un clarissime pour les filles. Cela avait plusieurs conséquences : le poids que pouvait avoir un préfet pour placer quelqu'un, la concurrence inévitable des réseaux entre eux, la chute de tout le réseau au moment de la disgrâce ou de la fin de fonction. Ce phénomène apparaissant clairement autour de Taruttienus sous Commode, mais il a dû se répéter sous une forme moins tragique à chaque changement de titulaire : un nouveau réseau peut se former. Le rôle important confié aux préfets tant comme chef de guerre qu'en tant que sommet et contrôle possible de l'administration, ainsi qu'en témoigne l'exemple de Saepinum, ou encore en relation avec l'instruction de procès, conduisait immanquablement à ce que les options personnelles du ou des préfets se retrouvent en partie dans la politique impériale. Leur réseau pouvait donc en partie recouper, au sein des élites impériales, des rassemblements d'opinions sur telle ou telle option politique. D'où l'impression d'un «parti de la guerre » rassemblé autour de Pompeianus et Tarrutienus en 180. Dernière conséquence il y avait nécessairement "personnalisation" de la fonction, et cela malgré la collégialité, d'autant plus qu’elle n’était pas aussi bien définie qu'une magistrature. Chaque préfet était un cas particulier et ses pouvoirs exacts dépendaient en grande partie finalement de la qualité des liens qu'il pouvait entretenir avec l'empereur et son éventuel collègue, le couple Perennis - Commode montrant jusqu'où pouvait aller ce rapport particulier entre l'empereur et les responsabilités confiées à son préfet. 
LES PREFETS DU PRETOIRE DE MARC AURELE

ANNEXE : récapitulatif des carrières connues pour les préfets du prétoire de Marc Aurèle

Sextus Cornelius Quirina Repentinus signo Contuccius

Aduocatus fisci

Procurator (C) [XX Hereditatium?]

Ab epistulis

Praefectus praetorio

Titus Furius Lucii filius Palatina Victorinus

Praefectus cohortis Bracarum in Britannia; Tribunus legionis II Adiutricis ; Praefectus alae Frontonianae

Procurator (C) prouinciae Galatiae; Procurator (CC) prouinciae Hispaniae; Procurator (CC) ludi magni ; Praefectus classis praetoriae Rauennatium ; Praefectus classis praetoriae Misenensium

Procurator a rationibus

Praefectus uigulum ; Praefectus Aegypti

Praefectus praetorio

Consularibus ornamentis honoratus et ob uictoriam Parthicam corona murali uallari aurea hastis puris IIII uexillis obsidionalibus IIII donatus

Titus Flauius Constans

Procurator $(C)$ prouinciae Daciae inferioris

Praefectus praetorio eminentissimus uir

Marcus Bassaeus Marci filius Stellatina Rufus

[Centurio] ; Primus pilus

Tribunus cohortis $V$ uigulum; tribunus cohortis $X$ urbanae; tribunus cohortis [...] praetoriae

Primus pilus bis

Procurator (CC) Asturiae et Callaeciae ; Procurator (CC) regni Norici ; Procurator (CC) prouinciarum Galliae Belgicae et duarum Germaniarum

Procurator a rationibus

Praefectus uigulum ; Praefectus Aegypti

Praefectus praetorio

Consularibus ornamentis honoratus et ob uictoriam germanicam et sarmaticam corona murali uallari aurea hastis puris IIII totidemque uexillis obsidionalibus donatus 
Marcus Macrinius [Claudia] Vindex

Procurator (CC) prouinciae Daciae Porolissensis

Praefectus praetorio

Publius Taruttienus Publii filius Poblilia Paternus

Ab epistulis latinis

Praefectus praetorio

[Consularibus ornamentis honoratus] ; adlectus inter consulares

Tigidius Perennis

[Praefectus annonae]

Praefectus praetorio 